\title{
Synaptic Depression and the Temporal Response Characteristics of V1 Cells
}

\author{
Frances S. Chance, Sacha B. Nelson, and L. F. Abbott \\ Volen Center and Department of Biology, Brandeis University, Waltham, Massachusetts 02254-9110
}

\begin{abstract}
We explore the effects of short-term synaptic depression on the temporal dynamics of $\mathrm{V} 1$ responses to visual images by constructing a model simple cell. Synaptic depression is modeled on the basis of previous detailed fits to experimental data. $A$ component of synaptic depression operating in the range of hundreds of milliseconds can account for a number of the unique temporal characteristics of cortical neurons, including the bandpass nature of frequency-response curves, increases in response amplitude and in cutoff frequency for transient stimuli, nonlinear temporal summation, and contrast-
\end{abstract}

The responses of cortical neurons to time-dependent stimuli display a paradoxical feature. In primary visual, auditory, and somatosensory cortices of rats, cats, and monkeys, many neurons exhibit responses to oscillatory stimuli that peak at frequencies of a few Hertz and fall rapidly to zero above $\sim 10 \mathrm{~Hz}$. This might give the impression that cortical neurons act as low-pass filters of the sensory stimuli that drive them. However, the same neurons can exhibit vigorous responses to transients, such as rapid stimulus onsets, that have much of their power above $10 \mathrm{~Hz}$. Why do these neurons respond to high-frequency stimuli that are novel and fail to respond to sustained stimuli over the same frequency range?

Neurons in the mammalian primary visual cortex (V1) display this paradoxical feature along with a number of other characteristics that reflect nonlinear temporal dynamics. Responses of V1 neurons fall off at lower temporal frequencies and at slower image velocities than those of neurons in the lateral geniculate nucleus (LGN) (Movshon et al., 1978; Orban et al., 1985; Hawken et al., 1996). Nevertheless, V1 neurons respond briskly to transients that contain high-frequency components, and responses to sustained stimuli are more transient than would be predicted on the basis of steady-state responses to oscillating images (Ikeda and Wright, 1975; Movshon et al., 1978; Kulikowski et al., 1979; Tolhurst et al., 1980). When visual images oscillating at different temporal frequencies are combined, V1 responses show nonlinear temporal summation. Even simple cells, which show approximately linear summation over different spatial regions of their receptive fields (Ferster, 1994), display nonlinear summation in the temporal domain. Combining two oscillating (counterphase) gratings with different temporal frequencies decreases the response to the more slowly oscillating image and enhances the response to the higher-

\footnotetext{
Received Jan. 8, 1998; revised March 27, 1998; accepted April 1, 1998.

This research was supported by the National Science Foundation Grants DMS95-03261 and IBN-95-11094, the W. M. Keck Foundation, National Eye Institute Grant EY-11116, and the Alfred P. Sloan Foundation.

Correspondence should be addressed to Dr. Larry Abbott, Volen Center, MS 013, Brandeis University, 415 South Street, Waltham, MA 02254-9110.

Copyright (C) 1998 Society for Neuroscience $0270-6474 / 98 / 184785-15 \$ 05.00 / 0$
}

dependent shifts in response phase. Synaptic depression also provides a mechanism for generating the temporal phase shifts needed to produce direction selectivity, and a model constructed along these lines matches both extracellular and intracellular data. A slower component of depression can reproduce the effects of contrast adaptation.

Key words: primary visual cortex; short-term plasticity; direction selectivity; contrast adaptation; simple cells; temporal summation

frequency oscillation (Dean et al., 1982). V1 cells respond to temporally irregular visual stimuli formed from sums of sinusoidal oscillations at temporal frequencies well above the response cutoff for simple sinusoidal oscillations (Reid et al., 1992). The phases of the responses of V1 neurons to temporally oscillating images shift as a function of contrast, again revealing temporal nonlinearity (Dean and Tolhurst, 1986; Carandini and Heeger, 1994). Nonlinear temporal dynamics is likely to contribute to a number of features exhibited by V1 cells, including direction selectivity (Reid et al., 1991; Jagadeesh et al., 1993; Tolhurst and Heeger, 1997) and velocity tuning (Orban et al., 1985). Intracellularly recorded membrane potentials from directionally selective neurons stimulated with sinusoidally oscillating gratings at certain spatial phases are distinctly nonsinusoidal (Jagadeesh et al., 1993). The mechanisms responsible for these temporal nonlinearities have not been identified.

In this paper, we explore the idea that short-term synaptic plasticity, in particular synaptic depression, is an important element in the nonlinear temporal dynamics that leads to enhancement of transient responses, nonlinear temporal summation, variable phase shifts, and direction selectivity. We also study the suggestion that a slow form of synaptic depression plays a significant role in contrast adaptation of V1 neurons (Nelson, 1991b; Finlayson and Cynader, 1995; Nelson et al., 1997; Todorov et al., 1997). Synaptic depression is a particularly prominent feature of transmission at neocortical synapses (Shaw and Teyler, 1982; Deisz and Prince, 1989; Thomson and West, 1993; Thomson et al., 1993). Short-term depression has been observed in studies of cat visual cortex (Stratford et al., 1996), in rodent somatosensory (Markram and Tsodyks, 1996; Tsodyks and Markram, 1997), motor (Thomson and Deuchars, 1994; Castro-Alamancos and Connors, 1996), and visual (Abbott et al., 1997; Varela et al., 1997) cortices, and at cat (Stratford et al., 1996) and rat (Gil et al., 1997) thalamocortical synapses. In our study of the dynamics of excitatory transmission from layer 4 to layer $2 / 3$ in slices of rat primary visual cortex (Abbott et al., 1997; Varela et al., 1997), we measured and modeled several components of short-term synap- 
tic plasticity acting over a number of time scales. Most prominent among these were two forms of synaptic depression: one rapid, setting in within 5-10 presynaptic action potentials and requiring 300-600 msec for recovery, and the other much slower, requiring many action potentials to reach full extent and recovering in $\sim 10$ sec. The recovery time for the rapid form of synaptic depression is in the correct range to contribute to the frequency dependence of cortical responses. It is nonlinear and, although suppressing sustained responses, can transiently transmit rapid stimulus onsets with high efficacy. The faster component of depression also acts on a timescale appropriate for contributing to response phase shifts, including those responsible for direction selectivity (Nelson et al., 1997).

Responses of V1 cells adapt to the level of contrast during prolonged visual stimulation (Movshon and Lennie, 1979; Ohzawa et al., 1985; Maddess et al., 1988; Bonds, 1991; Nelson, 1991a). The slower form of synaptic depression seen in the layer 4 to layer $2 / 3$ pathway seems well suited to contribute to this phenomenon. Its time course matches the time constants measured for contrast adaptation in single-unit and evoked-potential studies (Albrecht et al., 1984; Ho and Berkley, 1988; Maddess et al., 1988; Giaschi et al., 1993).

To examine the role of synaptic depression in shaping the temporal response properties of cortical neurons, we constructed a model of a V1 simple cell that receives its afferent drive via synapses that depress. Although the circuitry in the model is highly simplified, the synaptic dynamics is modeled quite accurately using a mathematical description that fits experimental data (Abbott et al., 1997; Varela et al., 1997). Although a number of mechanisms may contribute to temporal nonlinearities in cortical responses, we focus rather exclusively on synaptic depression within this modeling study to determine the limits of what it can explain and thereby to establish whether it is an important element in cortical dynamics.

\section{MATERIALS AND METHODS}

\section{The model}

Simple-cell model. The model simple cell that we study is a singlecompartment, integrate-and-fire neuron that receives synaptic input in the form of transient conductance changes at both excitatory and inhibitory synapses. The total excitatory and inhibitory synaptic conductances at time $t$ are denoted by $G_{E}(t)$ and $G_{I}(t)$ and are computed by summing contributions from all of the excitatory and inhibitory synapses, respectively. For convenience, we define synaptic conductances in dimensionless units so that $G_{E}$ and $G_{I}$ are the usual synaptic conductances divided by the resting membrane conductance of the cell. The membrane potential is computed by numerically integrating the first-order differential equation describing a resistance-capacitance (RC) circuit with additional synaptic conductances:

$$
\tau_{m} \frac{\mathrm{d} V}{\mathrm{~d} t}=V_{0}-V+G_{E}(t)\left(V_{E}-V\right)+G_{I}(t)\left(V_{I}-V\right)
$$

where $\tau_{m}$ equals $30 \mathrm{msec}$ and is the membrane time constant, $V_{0}$ equals $-70 \mathrm{mV}$ and is the resting potential, and $V_{\mathrm{E}}$ and $V_{\mathrm{I}}$ equal 0 and $-90 \mathrm{mV}$ and are the reversal potentials for the excitatory and inhibitory synapses, respectively. When the membrane potential reaches the threshold value of $-55 \mathrm{mV}$, an action potential is fired, and the membrane potential is reset to $-58 \mathrm{mV}$. The relatively high reset value was used to make the voltage traces match typical somatic recordings. Use of a lower value did not change the behavior of the model in any significant way.

When a presynaptic spike occurs on an excitatory afferent, the excitatory conductance is increased by the substitution:

$$
G_{E} \rightarrow G_{E}+g_{j} D_{j} S_{j}
$$

where $j$ is a label identifying which afferent fired. The parameter $g_{j}$ is a constant that sets the strength of synapse $j$, and $D_{j}$ and $S_{j}$ are factors describing its degree of fast and slow depression as discussed below. If the synapse for afferent $j$ is inhibitory, a similar increment is made in the inhibitory conductance:

$$
G_{I} \rightarrow G_{I}+g_{j} D_{j} S_{j}
$$

Between presynaptic action potentials, the synaptic conductances decay exponentially to zero with time constants $\tau_{E}=2 \mathrm{msec}$ and $\tau_{I}=10 \mathrm{msec}$ :

$$
\tau_{E} \frac{\mathrm{d} G_{E}}{\mathrm{~d} t}=-G_{E} \quad \text { and } \quad \tau_{I} \frac{\mathrm{d} G_{I}}{\mathrm{~d} t}=-G_{I} .
$$

Synaptic depression. Synaptic depression is modeled using the same formalism and parameter values used to fit slice data (Abbott et al., 1997; Varela et al., 1997). The general procedure is related to methods used previously to describe short-term plasticity at the neuromuscular junction (Liley and North, 1952; Magleby and Zengel, 1975; Krausz and Friesen, 1977; Zengel and Magleby, 1982; Sen et al., 1996) and adapted for our purposes (Abbott et al., 1997; Varela et al., 1997; see also Grossberg, 1984; Tsodyks and Markram, 1997). Each time a presynaptic action potential arrives at synapse $j$, the factors $D_{j}$ and $S_{j}$ for that synapse are reduced by multiplicative factors:

$$
D_{j} \rightarrow d_{j} D_{j} \quad \text { and } \quad S_{j} \rightarrow s_{j} S_{j} .
$$

The fixed parameters $d_{j}$ and $s_{j}$ (with $0 \leq d_{j}, s_{j} \leq 1$ ) determine the amount of depression at synapse $j$ induced by each spike and thereby control the depression onset rate. Between presynaptic action potentials, $D_{j}$ and $S_{j}$ recover exponentially toward the value one:

$$
\tau_{D} \frac{\mathrm{d} D_{j}}{\mathrm{~d} t}=1-D_{j} \quad \text { and } \quad \tau_{s} \frac{\mathrm{d} S_{j}}{\mathrm{~d} t}=1-S_{j}
$$

The time constants $\tau_{D}$ and $\tau_{S}$ determine the depression recovery rates. This model provides a good fit of experimental data (Varela et al., 1997). The two sets of equations listed above can be combined by writing:

$$
\tau_{D} \frac{\mathrm{d} D_{j}}{\mathrm{~d} t}=1-D_{j}+D_{j} \ln \left(d_{j}\right) \tau_{D} \sum_{\mu} \delta\left(t-t_{\mu}\right)
$$

and:

$$
\tau_{S} \frac{\mathrm{d} S_{j}}{\mathrm{~d} t}=1-S_{j}+S_{j} \ln \left(s_{j}\right) \tau_{S} \sum_{\mu} \delta\left(t-t_{\mu}\right),
$$

where $t_{\mu}$ is the time of a presynaptic spike labeled by the index $\mu$ and $\delta\left(t-t_{\mu}\right)$ is the Dirac $\delta$ function. These equations are convenient for determining average values of the depression factors, although this requires some care in taking the averages of the $\delta$ function terms. If afferent $j$ fires a Poisson spike train at rate $R_{j}$, the average steady-state values of the depression terms are:

$$
\left\langle D_{j}\right\rangle=\frac{1}{1+\left(1-d_{j}\right) \tau_{D} R_{j}} \quad \text { and } \quad\left\langle S_{j}\right\rangle=\frac{1}{1+\left(1-s_{j}\right) \tau_{S} R_{j}} .
$$

The parameter values we use lie within the range seen in the experimental data. For all of the simulations, $\tau_{D}=300 \mathrm{msec}$, and $\tau_{S}=20 \mathrm{sec}$. We use the values $s=0.99$ in all of the simulations where contrast adaptation is considered and $s=1.0$ when we simulate experiments involving a constant level of adaptation. (We write the depression factors $s_{j}$ and $d_{j}$ without the index $j$ when their values do not depend on $j$.) Turning off the slow form of depression in this way is a convenience to avoid having to duplicate the sorts of manipulations that must be done in an experimental setting to avoid adaptation effects. The value of $d$ varies between 0.4 and 1.0 in different simulations as noted.

The model of synaptic depression and parameters values used come from studies of slices of rat primary visual cortex. The data on visually evoked neural responses that we use to test the model come primarily from experiments on cats. Therefore, our model relies on two extrapolations, from in vitro to in vivo preparations and from rat to cat cortices. The extrapolation from rat to cat is supported by the fact that results on cat visual cortical synapses (Stratford et al., 1996) show short-term plasticity very similar to that seen in the rat. Measurements on slices of ferret visual cortex also reveal synaptic depression with similar properties (J. A. Varela and S. B. Nelson, unpublished observations). The relationship between short-term plasticity in vivo and in vitro has not been 
examined in detail in the visual system. However, synaptic depression has been observed in parallel in vivo and in vitro studies of rodent somatosensory cortex (Castro-Alamancos and Connors, 1996; Gil et al., 1997).

Synaptic inputs. To isolate the role that synaptic depression plays in shaping the temporal characteristics of V1 responses, we drive the model simple cell exclusively with feedforward inputs (Hubel and Wiesel, 1962). Although, in reality, V1 cells are part of a recurrent network, studying a feedforward model allows us to identify the essential features caused by synaptic depression without having to deal with the complexities of recurrent network dynamics. In a recurrent circuit, the nonlinear properties we study on the output of a model simple cell are also present on its inputs. To avoid this problem, we give all of the inputs to our model V1 cell, both excitatory and inhibitory, the spatial and temporal characteristics of linear LGN cells.

The model of synaptic depression we use is based on properties of the layer 4 to layer $2 / 3$ pathway within visual cortex that provides the major excitatory synaptic drive to upper layer neurons (Abbott et al., 1997; Varela et al., 1997). Recent results indicate that LGN inputs to neurons in the primary visual and somatosensory cortices display depression similar to that seen for synapses between pyramidal cells (Stratford et al., 1996; Gil et al., 1997). Currently, less information is available about the short-term synaptic plasticity of feedforward inhibition. Synapses from inhibitory interneurons onto pyramidal cells of rat primary visual cortex show the faster form of synaptic depression but to a lesser degree than excitatory synapses (Song et al., 1997). Unfortunately, we do not know how LGN-interneuron synapses contribute to the total short-term plasticity along the feedforward pathway. In the absence of these data, we simply assumed that the total synaptic depression of the inhibitory drive to the model V1 cell is the same as that of the excitatory drive. We also studied a feedforward model with exclusively excitatory drive. This model produced results similar to those described below, although, of course, there was no hyperpolarization below the resting potential.

The synaptic input to the model neuron is derived from the model of LGN center-surround receptive fields described in the next section. The structure of the receptive field of the model V1 simple cell is established by the spatial arrangement of the receptive fields of its on- and off-center afferents. Because we use contrast gratings that only vary spatially in one dimension as visual stimuli, we use a one-dimensional spatial arrangement of afferent receptive fields. To study response phase shifts, we consider a simple linear arrangement that produces a three-lobed, offon-off $\mathrm{V} 1$ receptive field. This is obtained by dividing the afferents of the model into three groups with receptive fields at three different spatial locations (see Fig. $2 A$ ). In the central region, we place on-center afferents that act on the model V1 neuron via excitatory synapses and off-center afferents that act via inhibitory synapses. In the two flanking regions, the situation is reversed so that the excitatory inputs have off-center receptive fields and the inhibitory inputs have on-center receptive fields. For the directionally selective model neuron, we use two such arrangements of afferent receptive fields shifted from each other by one-half the size of the receptive field center (see Fig. $3 A$ ). Because our primary purpose is to model the temporal response properties of V1 simple cells, we do not model spatial receptive fields in detail. We have obtained similar results from a variant of the model in which the spatial structure of the receptive field is matched to a Gabor function by adjusting the values of the synaptic weights of the inputs, but we use the simple geometric model here.

In the nondirectionally selective model (see Fig. $2 A$ ), all of the excitatory synaptic strengths were set to $g_{j}=0.009$, and the inhibitory synapses all had $g_{j}=0.0025$ (for exceptions, see Fig. $2 B$, middle and bottom, where values 2.4 and 10 times larger were used to compensate for the increased degree of depression). For the directionally selective model (see Fig. $3 A$ ), the nondepressing excitatory synapses had $g_{j}=0.0075$, and the nondepressing inhibitory synapses had $g_{j}=0.002$. For the depressing synapses, these values were increased by a factor of 10 . Some scaling of these values was done from figure to figure to produce firing rates within a range that matched data for a particular cell. In some cases (see Figs. 4-6), all of the synaptic conductances were multiplied by 1.25 . When we activated the slower form of synaptic depression to model contrast adaptation, we had to increase the synaptic conductances to compensate for the average tonic level of slow depression. The synaptic conductances were multiplied by factors of 5.5, 4, and 6.25 (see Figs. 7-9, respectively). Responses of the model without simulated visual images and using $g_{j}=$ 0.05 are also shown (see Fig. 1).

We use a sufficient number of afferents to drive our model V1 cell to reduce the Poisson noise in the membrane potential and the variability in the firing rate of the model simple cell to a level that allows us to perform single-trial simulations. This was done merely for convenience; using a smaller number of afferents and averaging over trials (as is done in experimental work) can yield similar results. In the receptive field, each circle represents 80 (see Fig. $2 A$ ) or 40 (see Fig. $3 A$ ) excitatory and inhibitory afferents. In another case (see Fig. 1), a total of 200 afferents was used.

Model of afferent firing rates. To model the afferent spike sequences that drive the V1 cell, we use a standard LGN model that produces a Poisson spiking output at a rate computed from a linear space-time filter acting on the luminance of the visual input (see, for example, Wörgötter and Koch, 1991). The particular implementation we use is from the work of Maex and Orban (1996). The spatial structure of the afferent receptive fields is center-surround described by the difference of two Gaussian functions. The temporal response of both the center and the surround is given by the difference of two $\alpha$ functions with the surround response slower than the center response. The difference between the stimulus luminance at the point $(x, y)$ at time $t$ and the average background luminance is denoted $I(x, y, t)$. The firing rate of afferent $j$ at time $t$ in response to this stimulus is given by the difference of center $(c)$ and surround $(s)$ contributions:

$$
\begin{aligned}
R_{j}(t)=R_{b} \pm A(C) \int \mathrm{d} x \mathrm{~d} y \mathrm{~d} t^{\prime} & {\left[W_{c}(x, y) K_{c}\left(t-t^{\prime}\right)\right.} \\
& \left.-0.6 \times W_{s}(x, y) K_{s}\left(t-t^{\prime}\right)\right] I\left(x, y, t^{\prime}\right) .
\end{aligned}
$$

$R_{b}$ is the background firing rate set to $5 \mathrm{~Hz}$ (for an exception, see Fig. 8 where we used a background rate of $15 \mathrm{~Hz}$ to get better agreement with the data), and $A(C)$ is a contrast-dependent amplitude factor discussed below with $C$ a measure of contrast that varies from zero to one. The choice of plus or minus in this equation determines whether the afferent is of the on-center $(+)$ or off-center $(-)$ type. The spatial filters for the center $(c)$ and surround $(s)$ are:

$$
W_{c, s}(x, y)=\frac{1}{2 \pi \sigma_{c, s}^{2}} \exp \left[-\frac{\left(x-x_{j}\right)^{2}+\left(y-y_{j}\right)^{2}}{2 \sigma_{c, s}^{2}}\right],
$$

where $\left(x_{j}, y_{j}\right)$ is the location of the center of the receptive field for afferent $j$. The receptive field size is set by the parameters $\sigma_{c}=0.3^{\circ}$ and $\sigma_{s}=1.5^{\circ}$. The temporal filters used to model the afferents are:

$$
K_{c, s}(t)=\alpha_{c, s}^{2} t \exp \left(-\alpha_{c, s} t\right)-\beta^{2} t \exp (-\beta t),
$$

with $1 / \alpha_{c}=8 \mathrm{msec}, 1 / \alpha_{s}=16 \mathrm{msec}$, and $1 / \beta=32 \mathrm{msec}$.

The computed firing rate $R_{j}$ is used to drive a Poisson spike generator that produces action potentials on afferent $j$ to the model V1 simple cell. The Poisson spike generator produces an action potential during a short time period of duration $\Delta t$ around the time $t$ with probability $R_{j}(t) \Delta t$. If $R_{j}(t)<0$, no spike is fired.

LGN firing rates do not increase linearly as image contrast is increased; they saturate. The afferent model described by the above equations is linear as a function of the stimulus function $I$, but we include this nonlinear effect via the contrast amplitude factor $A(C)$. To do this, we restrict $I$ to lie in the range $-1 \leq I \leq 1$. Afferent responses at contrast level $C$ are scaled by the contrast amplitude factor:

$$
A(C)=(172 \mathrm{~Hz}) \ln (67 C),
$$

obtained by fitting data of Ohzawa et al. (1985). We only use Equation 13 in the range $C>0.015$ (1.5\% contrast) where the logarithm is positive. To simulate images with no contrast, we set $A=0$. We have also used the fit $A \alpha C^{n} /\left(C_{50}^{n}+C^{n}\right)$ (Cheng et al., 1995) in the model and found that it gives similar results.

Comparison with data. We compare the results of the model with previously published data (see Figs. $3,4,7,8$ ). We have extracted the data curves or points in these figures from the cited references using the graph tracing program DataThief and then redrawn the figures.

\section{RESULTS}

\section{Transient responses}

The temporal response characteristics of the model simple cell we have constructed are affected by the temporal dynamics of the LGN cell model of the afferents and by synaptic depression. 
A
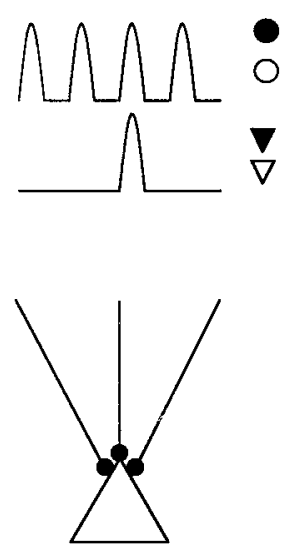

B

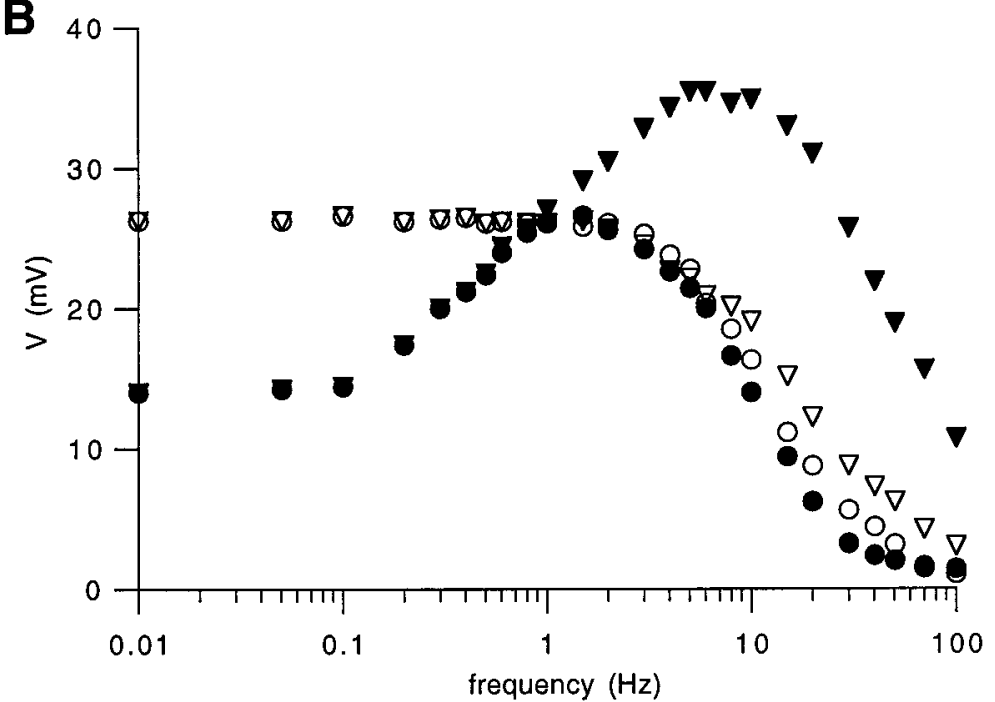

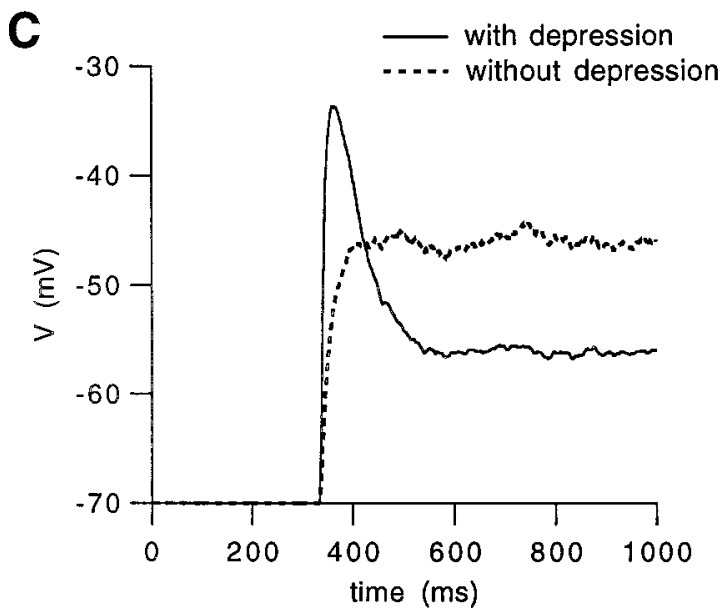

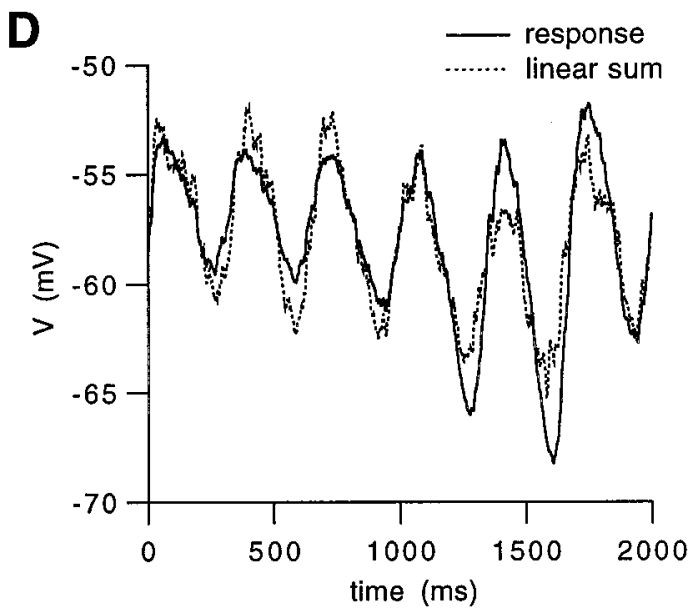

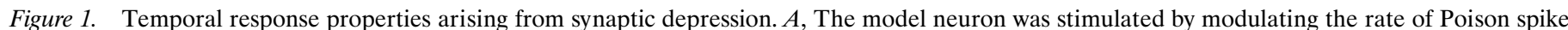

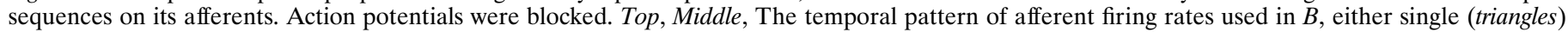

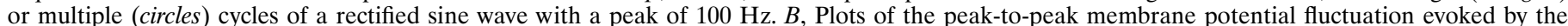

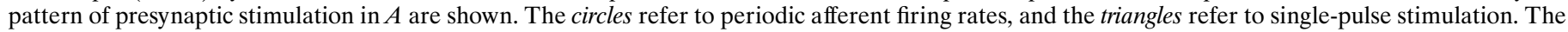

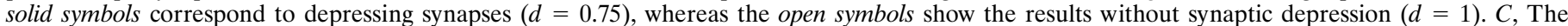

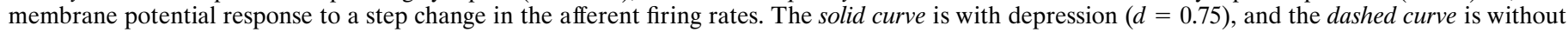

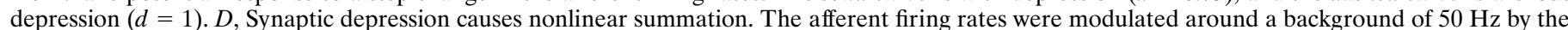

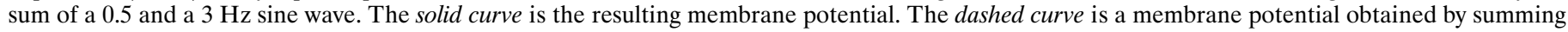

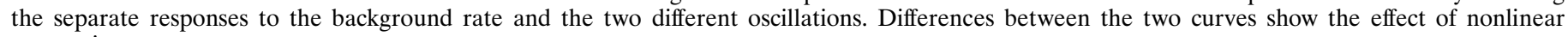
summation.

Nonlinear dynamics in the model V1 cell can arise from rectification of afferent and V1 firing rates as well as from nonlinear synaptic depression. Before analyzing the complete model, we present a simulation that reveals the basic features arising solely from the rapid form of synaptic depression. To isolate these features, we drive the model V1 neuron by manipulating directly the firing rate of its afferents rather than by using simulated visual images. Inhibitory afferents were not activated in this simulation. Furthermore, we study the membrane potential of the model simple cell with action potentials blocked. These steps eliminate the effects of temporal filtering by the LGN-like afferent model and of output firing rate rectification.

The frequency-response characteristics of the model V1 cell are shown in Figure $1 B$. To generate this figure, we modulated identically the firing rates of all the excitatory afferents to the model V1 cell, although individual action potentials were generated independently on each afferent. The common afferent firing rate oscillated over time at a variety of frequencies and took the form of a rectified sine wave with a peak firing rate of $100 \mathrm{~Hz}$. Two different cases were considered, a periodic pattern formed from a rectified sine wave as shown on the top of Figure $1 \mathrm{~A}$ and a single pulse consisting of one cycle of a rectified sine wave as shown in the middle of Figure $1 A$. Figure $1 B$ is a plot of the amplitude of the membrane potential fluctuations produced in the model V1 cell by these patterns of afferent firing. The solid circles and triangles correspond to the case in which the rapid form of depression was present with $d=0.75$. The open symbols show the results without any synaptic depression $(d=1.0)$ for compar- 
ison purposes. In both cases, the slow form of synaptic depression was eliminated by setting $s=1$. Conductance strengths were adjusted so that the peak responses to periodic input-rate oscillations were the same with and without synaptic depression.

For low afferent oscillation frequencies, the response amplitudes plotted in Figure $1 B$ for the periodic and transient cases are identical, but the frequency-response curves with and without depression are different. Without depression, the frequency response is approximately that of a resistance-capacitance circuit, and it peaks at zero frequency. With depression, the response peaks at $\sim 2 \mathrm{~Hz}$. The increase in response amplitude between 0 and $2 \mathrm{~Hz}$ is attributable to the onset of depression during the rising phase of the afferent firing rate increase. At low modulation frequencies, depression sets in well before the afferent rate reaches its peak value. At higher frequencies, the afferent firing rate increases quickly enough so that it can get closer to its peak value before depression sets in. The peak response frequency depends on the value of $d$, and for typical values seen in the slice data (Varela et al., 1997), such as $d=0.75$, it falls in a range that matches peak response frequencies for $\mathrm{V} 1$ neurons responding to oscillating images. Above a few Hertz, the response amplitude for single pulse afferent rates without depression and for periodic afferent rates with and without depression all fall off rapidly as a function of frequency. This is the result of the low-pass filtering properties of the equivalent circuit model of the neuron [for a discussion of a bandpass filtering model, see Maex and Orban (1992)]. Synaptic depression causes the response to periodic afferent rate fluctuations to roll off slightly more rapidly than when depression is absent. This is because, for high-frequency oscillations, there is insufficient time for the synapses to recover from depression between successive pulses of afferent firing. The solid triangles in Figure $1 B$ show that synaptic depression has a dramatic effect on responses to transient, single-pulse fluctuations in the afferent firing rates. For such transients, the response amplitude continues to rise as a function of frequency until it peaks at $\sim 10 \mathrm{~Hz}$. This occurs because, for single pulses, recovery from depression between pulses is not an issue and the rapid onset of a high-frequency pulse allows the firing rate to get closer to its maximum value before depression sets in. The eventual roll off above $10 \mathrm{~Hz}$ is due to the filtering properties of the postsynaptic cell. The effects of synaptic depression seen in Figure $1 B$ reproduce characteristic features of cortical responses: the rise in response amplitude at low frequency, the response peak at a few Hertz, and the increase in both response amplitude and response cutoff frequency for transient as opposed to periodic stimuli.

Figure $1 C$ shows the membrane potential of the model neuron in response to a sudden step in the afferent firing rates from 0 to $50 \mathrm{~Hz}$. The dashed curve, showing the response without synaptic depression, resembles a typical capacitive charging curve. When synaptic depression is included, the membrane potential overshoots by approximately a factor of two and then settles to its steady-state value. Similar overshoots are a common feature of the firing rates of cortical neurons in response to sudden stimulus onsets (Maunsell, 1987).

The reduced model, in which synaptic depression is the only nonlinearity, displays nonlinear temporal summation as seen in Figure $1 D$. The membrane potential trajectory indicated by the solid line in Figure $1 D$ was evoked by setting all of the excitatory afferent firing rates to an expression involving the sum of two sinusoids: $r=50 \mathrm{~Hz}\left[1+0.5 \sin \left(2 \pi f_{1} t\right)+0.5 \sin \left(2 \pi f_{2} t\right)\right]$, with $f_{1}$ $=0.5 \mathrm{~Hz}$ and $f_{2}=3 \mathrm{~Hz}$. The dashed line in Figure $1 D$ was computed by summing the membrane potential trajectories gen- erated by the DC term and the two sinusoids in this expression separately. The failure of linear summation is evident. The response of the model to a combined stimulus like this, consisting of a sum of low- and high-frequency oscillations, can be separated into corresponding low- and high-frequency components by Fourier analysis. When this is done, we find that in the model, as in the data (Dean et al., 1982), the amplitude of the low-frequency component is smaller than when the low-frequency signal is presented alone, whereas the amplitude of the high-frequency component is larger than when the high-frequency stimulus is presented alone. This latter effect can be seen by carefully comparing the two traces in Figure $1 D$. This figure also reveals that the low-frequency oscillation induces more of a multiplicative amplitude modulation in the response to the high-frequency stimulus than a linear additive shift.

We can use the result in Equation 9 to understand the nonlinear summation seen in Figure $1 D$. Suppose that $R=A+B \sin (2 \pi$ $\left.f_{1} t\right)+C \sin \left(2 \pi f_{2} t\right)$ as in Figure $1 D, f_{1}$ is small enough so that the depression factor $D$ remains near its steady-state value during the slower oscillations, and $f_{2}$ is large enough so that oscillations at this frequency have a minimal temporal effect on synaptic depression. In this case, $D \approx r_{D} /\left[r_{D}+A+B \sin \left(2 \pi f_{1} t\right)\right]$, where $r_{D}=$ $1 /\left[(1-d) \tau_{D}\right]$. The level of postsynaptic drive attributable to an afferent firing at rate $R$ via a synapse depressed to a level $D$ is approximately $D R$. Thus, the effect of these two different sinusoids is approximately $r_{D}\left[A+B \sin \left(2 \pi f_{1} t\right)+C \sin \left(2 \pi f_{2} t\right)\right] /\left[r_{D}\right.$ $\left.+A+B \sin \left(2 \pi f_{1} t\right)\right]$. The rapidly varying part of this expression, $r_{D} C \sin \left(2 \pi f_{2} t\right) /\left[r_{D}+A+B \sin \left(2 \pi f_{1} t\right)\right]$, shows the effect seen in Figure $1 D$, in which the amplitude of the high-frequency oscillation is modulated by a low-frequency term in a multiplicative rather than in an additive form.

\section{Temporal phase shifts}

Having illustrated the basic features introduced by synaptic depression, we now include the LGN-like afferent model in our simulations and drive the model V1 neuron with simulated visual input. The basic arrangement considered in this section is shown in Figure $2 A$. The afferent receptive fields are arranged to form a V1 receptive field consisting of a central "on" region and two flanking "off" regions. The visual image used in these simulations is a grating with sinusoidal variations in both space and time. The maximum contrast and temporal oscillation frequency of the grating were varied, but its spatial wavelength was held fixed at a value that maximized the V1 response.

The membrane potential of the model neuron, with action potentials blocked, in response to an oscillating grating with different degrees of depression is shown in Figure $2 B$. Without depression $(d=1.0)$, the membrane potential varies approximately sinusoidally because the transform from image luminance to membrane potential is approximately linear in the model. When depression is included ( $d=0.75$ and $d=0.4$ ), a temporal nonlinearity is immediately evident because the waveform in response to a sinusoidal input is not sinusoidal. The time of peak depolarization (and peak hyperpolarization) advances via the effects of synaptic depression until it shifts by almost $90^{\circ}$. This occurs because depression sets in during the rising phase of the afferent response. The magnitude of the phase shift depends on both the frequency and the level of contrast. To quantify this effect, we computed the phase of the Fourier component of the membrane potential at the frequency of the stimulus. This is plotted as a function of stimulus frequency in Figure $2 C$. The response phase of a resistance-capacitance circuit varies as a 
A

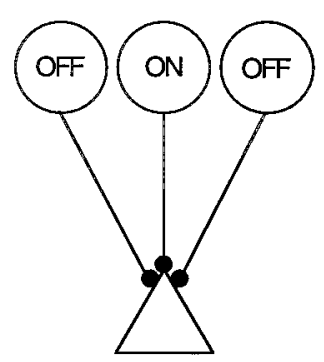

B
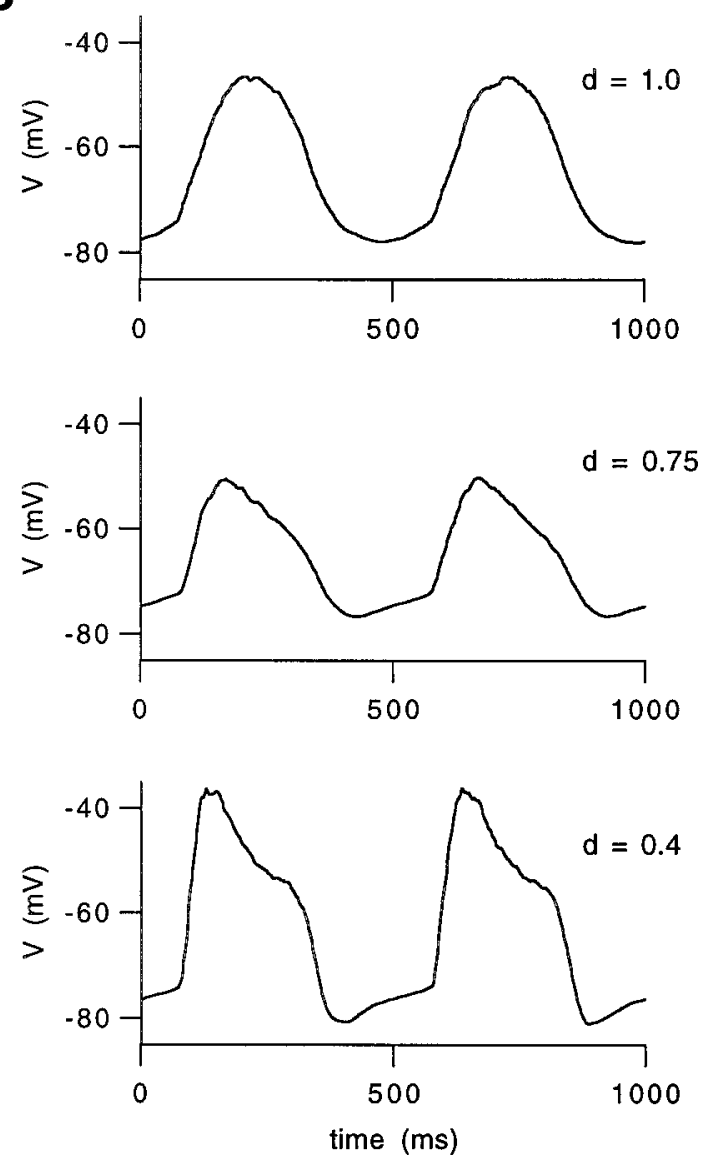

C

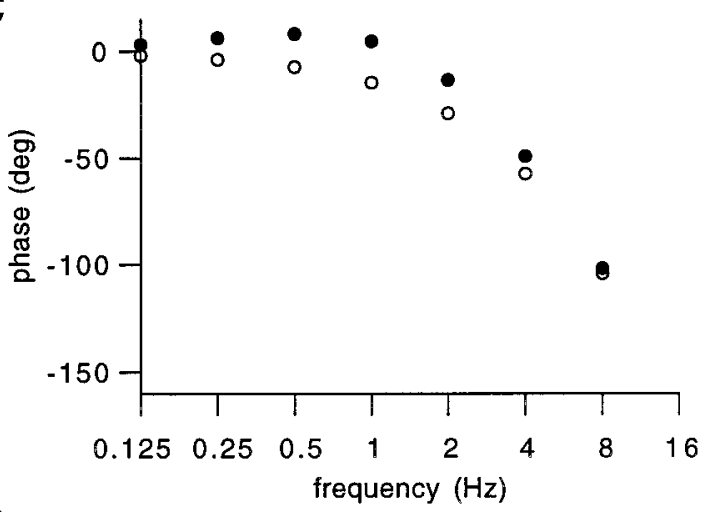

D

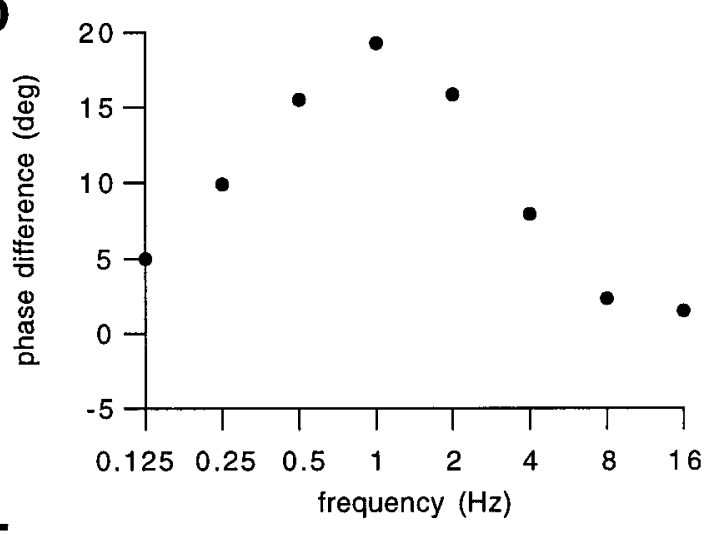

$\mathbf{E}$

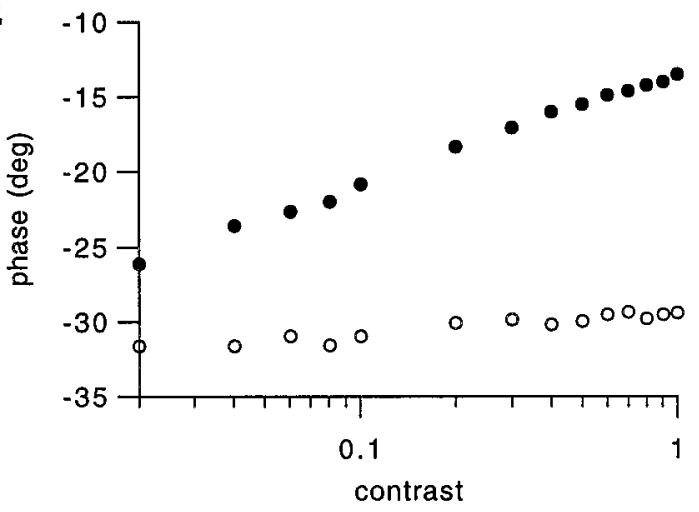

Figure 2. Synaptic depression causes temporal phase shifts. $A$, Schematic of the arrangement of afferent receptive fields used to drive the model simple cell. Areas marked OFF correspond to the central regions of off-center afferents with excitatory synapses onto the simple cell and of on-center afferents with inhibitory synapses. Conversely, within the ON region, the central regions of on-center afferents excite the model V1 cell, and of off-center afferents inhibit it. For $B-E$, the stimulus used was a contrast grating that varied as $\sin (2 \pi f t) \sin (2 \pi x / \lambda)$, where $f$ is the temporal frequency and $\lambda$ is the spatial wavelength. The spatial wavelength was set to match the spacing of the afferent receptive fields, whereas the temporal oscillation frequency or contrast was varied. $B$, Membrane potential as a function of time in response to a $2 \mathrm{~Hz}$ oscillating grating (100\% contrast) for different levels of synaptic depression. Top, no depression; middle, $d=0.75$; bottom, $d=0.4$. $C$, Response phase relative to the phase of the oscillating grating for different oscillation frequencies at $100 \%$ contrast. Open circles correspond to no depression, and solid circles correspond to $d=0.75$. $D$, The difference between the response phases in $C$ with and without synaptic depression plotted as a function of stimulus frequency. $E$, The response phase relative to the stimulus for an oscillation frequency of $2 \mathrm{~Hz}$ as a function of stimulus contrast. Open circles correspond to no depression, and solid circles correspond to $d=0.75$.

function of frequency and can account for the major part of the dependence of response phase on frequency. However, depression produces an appreciable phase advance for frequencies between $\sim 0.25$ and $6 \mathrm{~Hz}$ relative to the phase of the nondepressing case (Fig. $2 C$, open circles). The phase advance attributable to depression is plotted in Figure $2 D$. Note that the phase shift computed in this way is considerably smaller than the phase shift of the peak of the membrane potential depolarization seen in Figure $2 B$.
For a linear system, the phase cannot depend on amplitude or, in this case, contrast. When synaptic depression is eliminated, the phase of the model V1 cell membrane potential oscillations is indeed insensitive to contrast (Fig. 2E, open circles). Depression causes the phase to advance as the contrast is increased (Fig. $2 E$, solid circles). Phases of experimentally recorded V1 responses advance as the contrast is increased in qualitative agreement with the results of Figure $2 E$, but the magnitude of the effect is considerably larger in the data (Dean and Tolhurst, 1986; Ham- 
ilton et al., 1989; Reid et al., 1991; Carandini and Heeger, 1994). There are some complications in relating the phase of the membrane potential to the phase extracted from the principal Fourier component of a spike sequence. As we have mentioned, the peak phase of the membrane potential advances more than the phase of its principal Fourier component. The phases extracted from spikes, from the peak potential, and from subthreshold oscillations are all different, and the relationship between them depends on details of the membrane potential waveform and on the value of the spiking threshold. Synaptic depression is only one potential contributor to response phase shifts. Additional LGN shifts, spike-rate adaptation, conductance changes, and intracortical effects are other possible sources (Priebe et al., 1997; Carandini et al., 1998).

\section{Direction selectivity}

Models of direction selectivity in neuronal responses to moving images are based on a combination of temporal and spatial phase shifts (Barlow and Levick, 1965; Adelson and Bergen, 1985; Watson and Ahumada, 1985; Borst and Egelhaaf, 1989; Heeger, 1993; Smith and Snowden, 1994; Suarez et al., 1995; Maex and Orban, 1996). In these models, inputs from different spatial locations within the receptive field are subjected to different temporal delays or advances so that an image moving in the preferred direction produces synchronous excitation and a large response. Movement in the opposite direction leads to asynchronous excitation and a weaker response. A spatial phase shift between different sets of inputs to a V1 neuron can be established by appropriate positioning of the receptive fields of its afferents. The source of the temporal phase shift is more problematic. Potential sources include lagged responses in the LGN (Mastronarde, 1987; Saul and Humphrey, 1990) or delays caused by cortical loops (Suarez et al., 1995; Maex and Orban, 1996). In both of these cases, the relevant temporal phase shift is a delay. Figure $2 B$ shows that synaptic depression is another candidate, although in this case the temporal phase shift is an advance not a delay.

Figure 3 shows how the phase advance attributable to synaptic depression can give rise to directionally selective responses. The key element in the model is a correlation between the spatial location of a given input and the degree of depression it displays. We discuss arrangements with multiple components and graded degrees of depression below, but first we consider the simplest possible scheme. As seen in Figure $3 A$, the model V1 neuron is driven by two sets of inputs each consisting, as before, of a central on region flanked by two off regions. The two sets are shifted in space relative to each other by one-half the size of the central region of the afferent receptive field. This produces the spatial shift needed in the model. The temporal shift is generated by connecting these two sets of inputs to the model cortical neuron via synapses exhibiting different degrees of depression. In this example, one set has no depression $(d=1)$, and the other has a fairly large amount of depression $(d=0.4)$. The membrane potential fluctuations produced in the model V1 cell (with action potentials blocked) by these two sets of inputs separately are shown on the left of Figure $3 B$. These traces were generated by a temporally oscillating, stationary grating positioned to produce the maximum response in each case. The set of nondepressing inputs evokes an approximately sinusoidal oscillation (dashed curve) like that seen in the top of Figure $2 B$. Input through the depressing synapses generates a sawtooth waveform (solid curve) similar to that seen in the bottom of Figure $2 B$. The sawtooth waveform is advanced in phase relative to the sinusoidal form.
Intracellular recordings of direction-selective neurons in cat visual cortex have been made by Jagadeesh et al. (1993). We compare the membrane potential of our model neuron with their results in Figure 4. Kontsevich (1995) (see also Ferster, 1994) has analyzed this intracellular data and discovered that it can be fit quite well using a phenomenological model based on two principal components. The components extracted by Kontsevich (Fig. $3 B$, right) are quite similar to the two components that contribute to the membrane potential in our model (Fig. 3B, left). This similarity first led us to consider synaptic depression as a mechanism for direction selectivity.

Figure 3, $C$ and $D$, shows how the model of direction selectivity works. When a grating moves in the preferred direction (Fig. $3 A$, from left to right), it aligns with the receptive fields of the afferents with nondepressing synapses before it aligns with those that depress. Nevertheless, synaptic depression advances the response so that the peak of the depolarization caused by the depressing synapses (Fig. 3C, upper, solid curve) occurs at approximately the same time as the peak of the contribution of the nondepressing synapses (Fig. 3C, upper, dashed curve). When the two contributions are added together, they produce sufficient depolarization to make the model neuron fire action potentials (Fig. 3C, lower). When the direction of motion is reversed, the grating aligns with the nondepressing inputs after it aligns with the receptive fields of the depressing synapses. In this case, the contribution of the depressing synapses to the membrane potential (Fig. 3D, upper, solid curve) is out of phase with that of the nondepressing synapses (Fig. 3D, upper, dashed curve). When the two are summed, the total depolarization is subthreshold, and no firing results (Fig. $3 D$, lower).

Figure 4 compares the membrane potential in our model with the intracellular recordings of Jagadeesh et al. (1993). A stationary oscillating grating was used as the stimulus for both the model and the experiments, and the different traces show the responses for different positions of the grating. In both cases, the response at $0^{\circ}$ spatial phase is approximately sinusoidal. In the model, this occurs because the grating in this position aligns with the receptive fields of the inputs without synaptic depression. At approximately $90^{\circ}$ spatial phase, the membrane potential in both the model and the data has the sawtooth shape that we have seen before. In the model, this occurs because the grating now aligns with the receptive fields of the afferents with synapses that depress.

Any model of direction selectivity must account for the fact that neurons can remain directionally selective over a wide range of contrasts. Figure $5 A$ shows that this is true for our model. The firing rate of the model V1 neuron increases as a function of contrast for motion in both the preferred and nonpreferred directions. However, the direction index is constant and near one over the entire contrast range shown (the direction index is the difference between the firing rates in the preferred and nonpreferred directions divided by the firing rate in the preferred direction). Figure $5 B$ shows that the firing rate evoked by a grating moving in the preferred direction varies with temporal frequency in the typical manner of a cortical response, peaking at $\sim 2 \mathrm{~Hz}$ and falling off above $\sim 10 \mathrm{~Hz}$ (for a moving grating, the temporal frequency is the speed of the motion divided by the spatial wavelength of the grating). Although the response in the preferred direction is greatly decreased at low temporal frequencies, the model neuron remains at least somewhat directionally selective. Directionally selective neurons show a wide variety of frequency and contrast-response characteristics, and those shown 

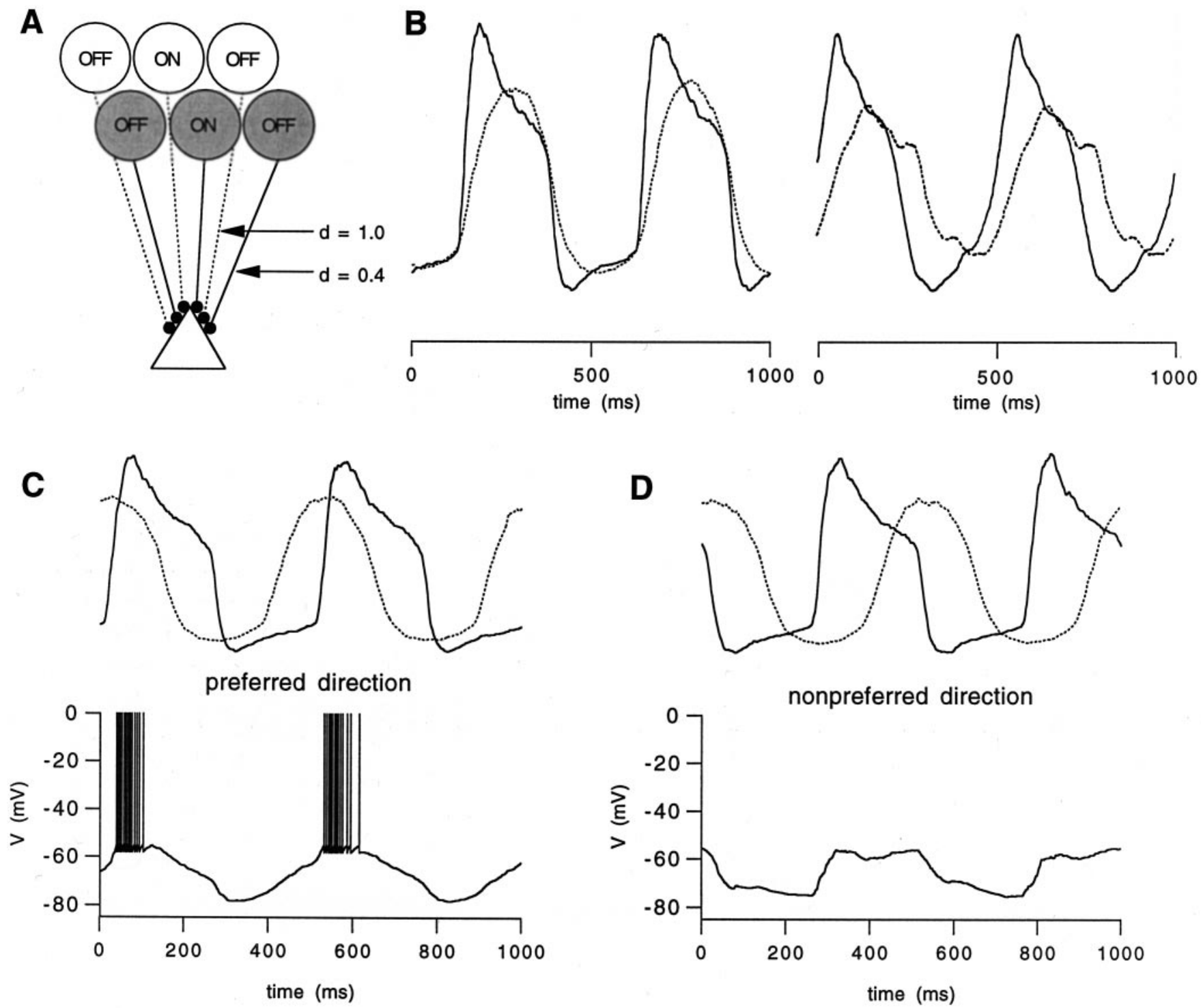

Figure 3. Model of a directionally selective simple cell. A, The arrangement of afferent receptive fields. Each row of circles is identical to that shown in Figure $2 A$, and the two rows are displaced from each other in the horizontal direction by one-half the width of the central region of an afferent receptive field (the vertical displacement of the two rows in the figure is only for clarity; the V1 receptive field is one-dimensional). The upper circles represent afferents coupled to the V1 cell without synaptic depression, and the lower circles are inputs with synapses that depress $(d=0.4)$. B, Left , Model V1 cell membrane potentials evoked by driving the two rows of inputs separately using a sinusoidal counterphase grating oscillating at $2 \mathrm{~Hz}$. The solid curve is the potential caused by driving the afferents with depressing synapses (shaded circles in $A$ ), and the dashed curve corresponds to driving the afferents with nondepressing synapses (open circles in $A$ ). Right, The two principal components replotted from Kontsevich's (1995) analysis of the intracellular recordings of Jagadeesh et al. (1993). $C$, Plots of the membrane potential of the model simple cell in response to a grating moving in the preferred direction. Upper, The peak membrane potential fluctuations induced separately by the depressing (solid curves) and nondepressing (dashed curves) inputs are in phase. Lower, When both components are present, the model neuron fires action potentials. $D$, Plots of the membrane potential of the model simple cell in response to a grating moving in the nonpreferred direction. Upper, The membrane potential fluctuations induced separately by the depressing (solid curves) and nondepressing (dashed curves) inputs are out of phase. Lower, When both components are present, the model neuron fails to fire any action potentials.

in Figure 5 fall within observed ranges (Orban et al., 1986; Reid et al., 1991; Tolhurst and Dean, 1991; Saul and Humphrey, 1992).

The directionally selective model of Figures 3-5 is based on having two populations of inputs with different spatial phases and different degrees of depression. It is possible to avoid such a simplistic scheme and construct models that involve a continuous variation in the degree of synaptic depression. The critical component in these models is that the degree of depression for a given input must be correlated with its spatial phase. We have constructed such a model with spatial receptive fields divided into two groups, as in Figure $3 A$, but with a continuous range of depression factors. These factors fell in the range $0.4 \leq d_{j} \leq 1.0$. Afferents with $d_{j} \leq 0.7$ shared one spatial placement, and those with $d_{\mathrm{j}}>0.7$ had the second spatial placement. The firing rates, direction index, and frequency-response curves for this model are shown in Figure 6 . The graded model tends to be less directionally selective than the simple two-component model, although it retains a constant directional index as a function of contrast (Fig. 6A). The difference in the frequency-response profile of this model neuron (Fig. $6 B$ ) for motion in the nonpre- 
A

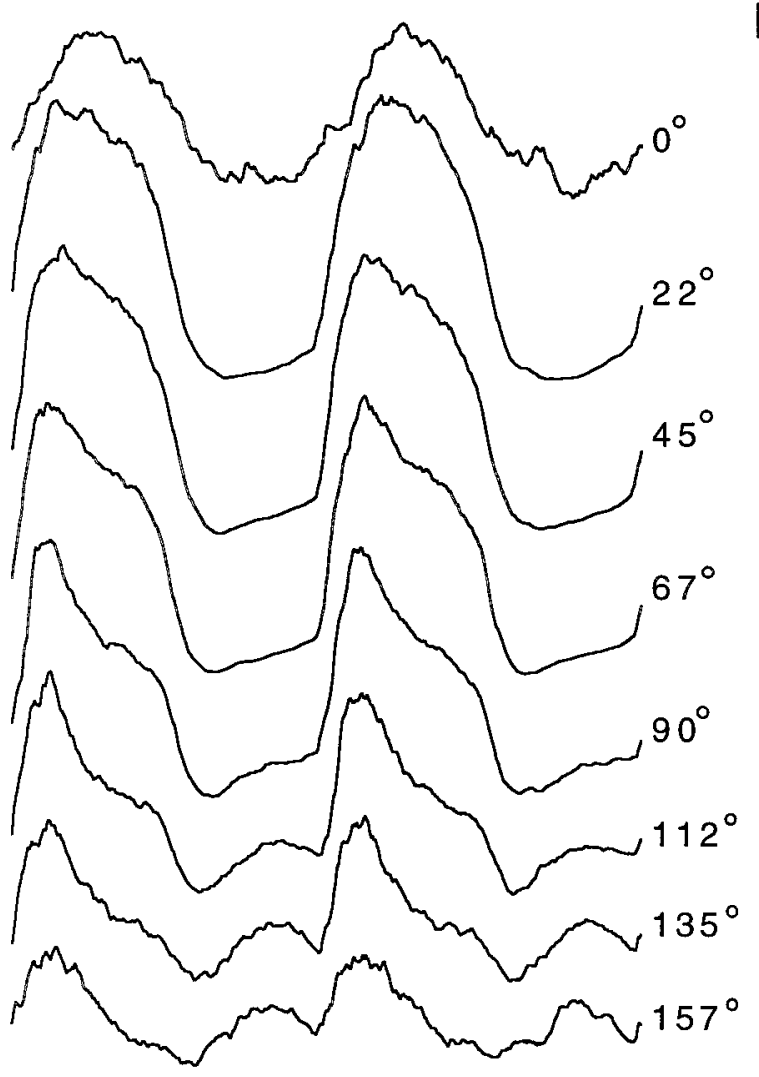

B

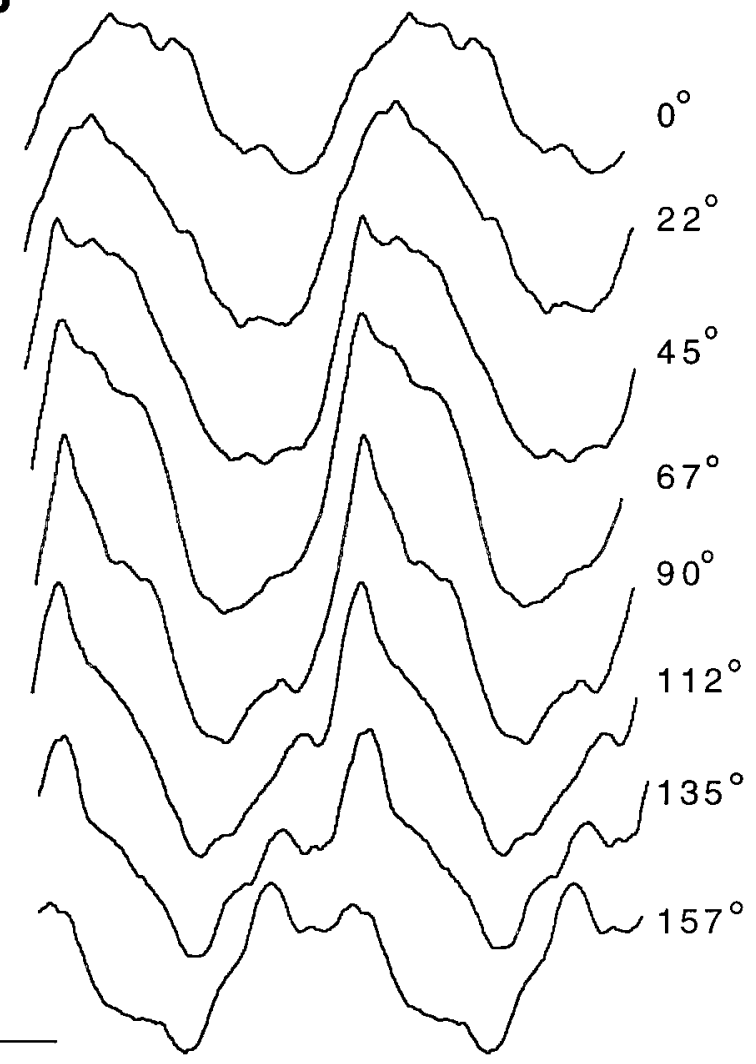

Figure 4. A comparison of the membrane potential of the model simple cell with in vivo intracellular recordings from a neuron in area 17 of a cat. In both cases, the stimulus was a stationary counterphase grating oscillating at $2 \mathrm{~Hz}$ and positioned at different spatial phases as indicated. $A$, Membrane potential of the model neuron. $B$, Recorded membrane potentials replotted from Jagadeesh et al. (1993). Calibration: horizontal, 100 msec; vertical, 10 $\mathrm{mV}$ for the model and $2.5 \mathrm{mV}$ for the data.

A

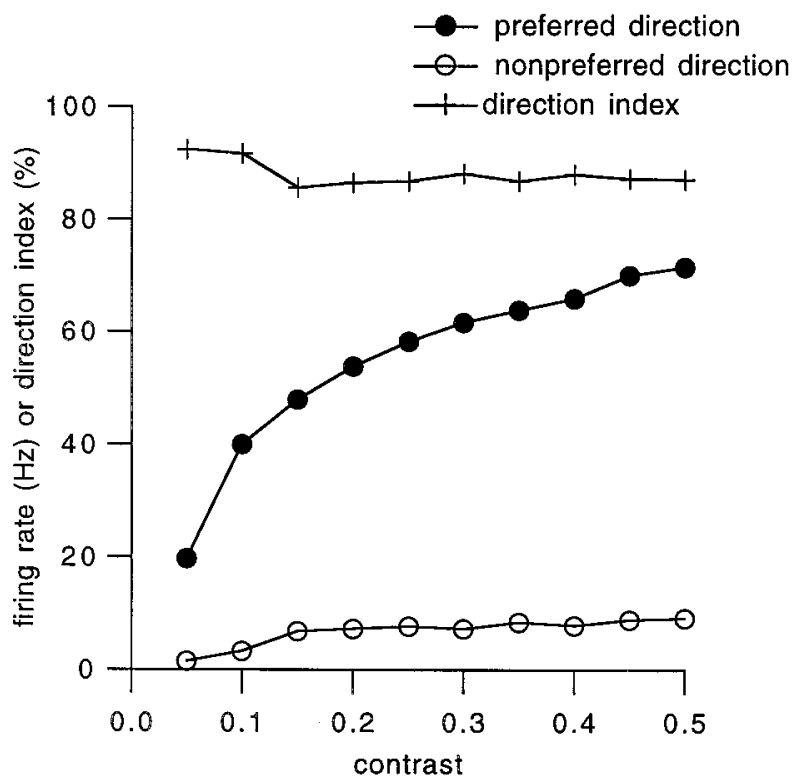

B
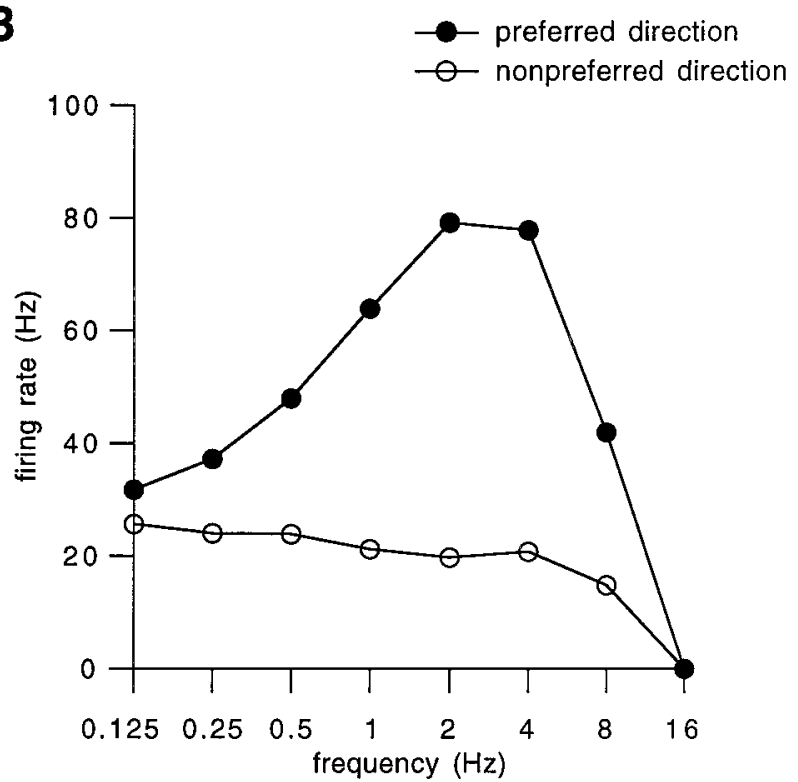

Figure 5. Direction selectivity as a function of contrast and frequency. $A$, The firing rate of the model V1 cell shown in Figures 3 and 4 as a function of the contrast of the moving grating for motion in the preferred (solid circles) and nonpreferred (open circles) directions. The upper curve (+ symbols) is the directional index, plotted as a percentage. $B$, The firing rate in response to a grating of $100 \%$ contrast moving in the preferred (solid circles) and nonpreferred (open circles) directions as a function of frequency. 
A

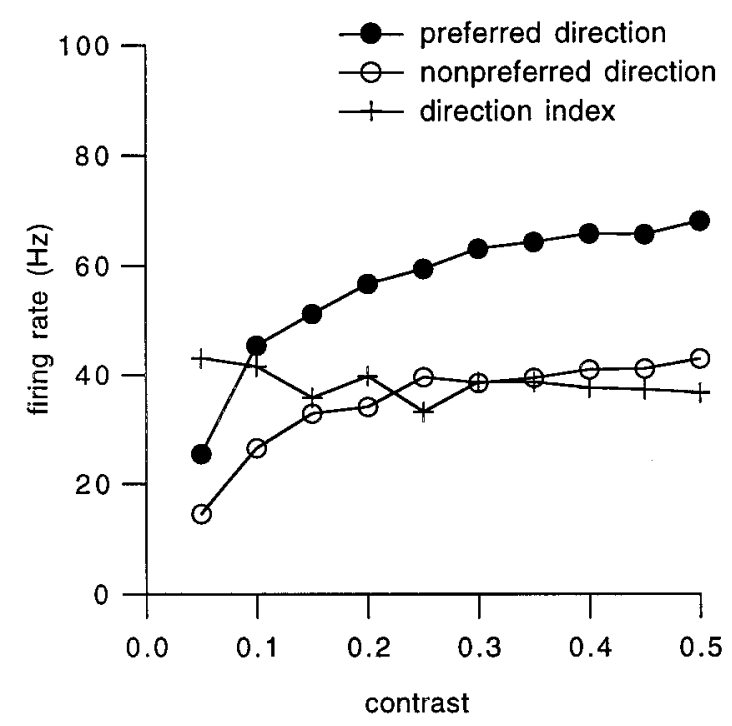

B

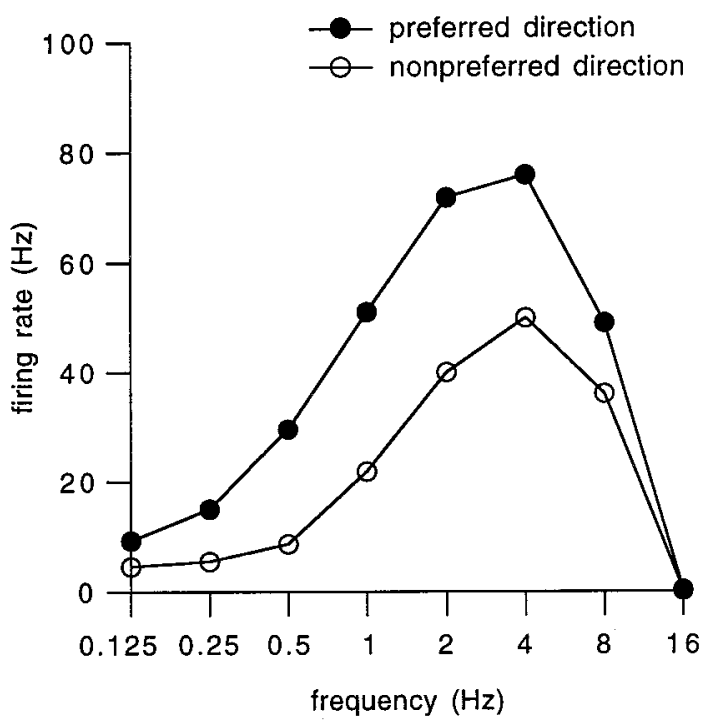

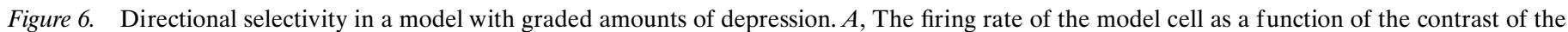

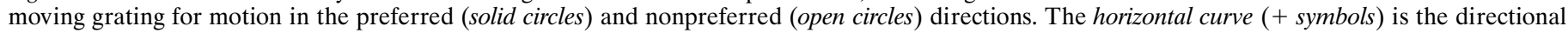

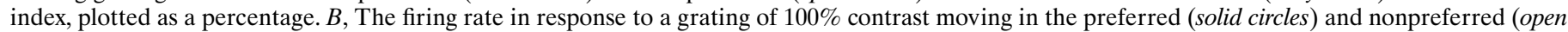
circles) directions as a function of frequency.

ferred direction compared with that in Figure $5 B$ is attributable primarily to its higher firing rate compared with that of the model of Figure $3 A$ and is not a necessary correlate of this architecture.

We have used oscillating stimuli and discussed phase shifts in our presentation of the directionally selective model, and that might leave the impression that it requires oscillating stimuli to be directionally selective. We have verified that this is not the case. For example, the response of the model neuron to two spatially displaced gratings that are flashed transiently depends on the order in which they appear in a manner consistent with its direction selectivity.

\section{Contrast adaptation}

The effects we have discussed thus far are caused by the fast component of synaptic depression. We now turn our attention to the slower component that, when activated by setting $s=0.99$ (rather than $s=1$ ), causes the responses of the model simple cell to change slowly over time as the slow depression either sets in or recovers from previous activity. Figure 7 compares a model simulation with an experiment on contrast adaptation in neurons of cat area 17 performed by Ohzawa et al. (1985). The stimulation sequence in Figure 7 goes from top to bottom. In both the simulation and the experiment, the firing rate at $0 \%$ contrast is essentially zero. After a $30 \mathrm{sec}$ period during which contrast adaptation to the $0 \%$ stimulus takes place, the response to a low-contrast grating is transiently vigorous but then relaxes to a lower firing rate. After another $30 \mathrm{sec}$ adaptation period, the response to a high-contrast grating once again consists of a transient period of rapid firing followed by steady-state firing at a lower rate. When the low-contrast grating is presented for a second time, there is no initial response because of the previous adaptation to the high-contrast stimulus. The steady-state response is established after a slow buildup period. The model neuron, with contrast adaptation arising solely from slow synaptic depression, duplicates the results seen in the experimental recordings quite well.
Figure 8 compares the contrast-response curves for the model neuron and a cat area 17 neuron recorded by Ohzawa et al. (1985) under a variety of preadapted conditions. Contrast adaptation causes the curves to shift rightward in the model as in the data. We can approximate the effect of synaptic depression on contrast response using some of the results derived when we discussed the model of depression. The total drive coming from an afferent with firing $R$ is $R$ times the level of depression of its synapse onto the V1 neuron. The average steady-state level of depression is given by Equation 9. After adaptation to a sustained firing rate $R_{\text {adapt }}$, this drive is equal to $R /\left(\left[1+\left(1-d_{j}\right) \tau_{D} R\right][1+(1-\right.$ $\left.\left.s_{j}\right) \tau_{S} R_{\text {adapt }}\right]$ ). Synaptic depression thus implements a form of divisive contrast normalization similar but not identical to that discussed by Heeger (1992) and Carandini and Heeger (1994). Synaptic depression causes the postsynaptic response to begin to saturate when the input rates are larger than $r_{D}=1 /\left[(1-d) \tau_{D}\right]$. In agreement with experimental data (Albrecht and Hamilton, 1982; Skottun et al., 1986), the saturation level depends on the afferent firing rate and hence on the contrast level, not on the firing rate of the postsynaptic V1 neuron.

Recently two groups have recorded intracellularly in anesthetized cats during contrast adaptation protocols (Ahmed et al., 1997; Carandini and Ferster, 1997). Ahmed et al. (1997) found no clear intracellular correlate of contrast adaptation in the recorded subthreshold membrane potentials. Carandini and Ferster (1997) have reported that the dominant effect of contrast adaptation on the recorded membrane potentials was a DC hyperpolarization. In the majority of cells, contrast adaptation did not significantly alter the amplitude of the membrane potential oscillations evoked by an oscillating stimulus. Our model is not in complete agreement with these results. Figure 9 shows the membrane potential of the model simple cell in response to oscillating gratings at 0,5 , and $100 \%$ contrast. The solid curves show the response after adaptation to a $100 \%$ contrast grating, and the dashed curves show the response after adaptation to a 5\% contrast grating. Adapta- 

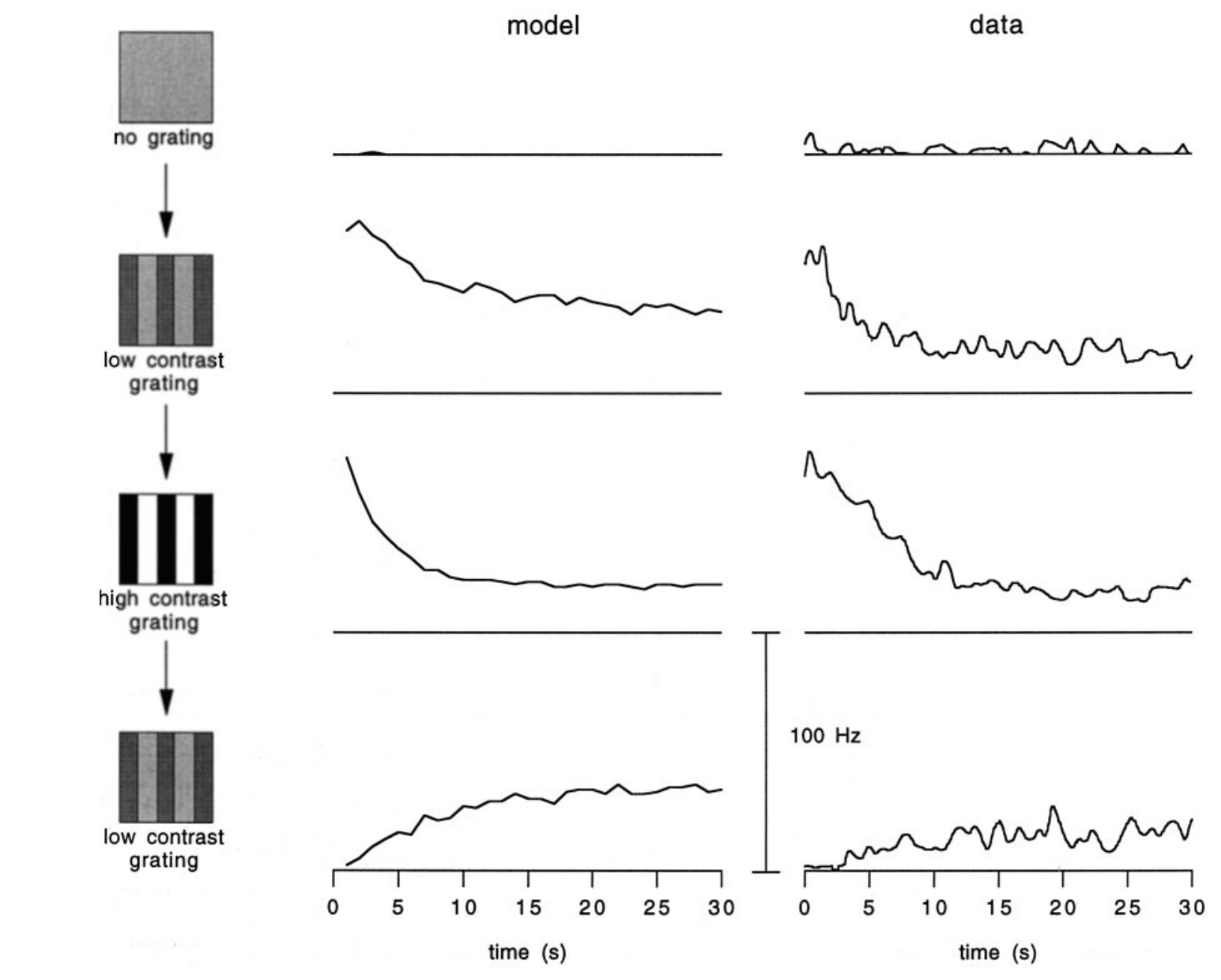

data

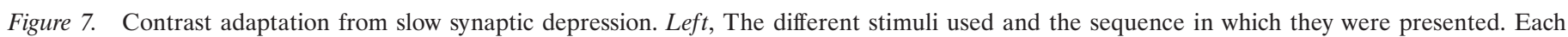

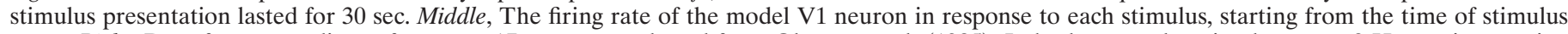

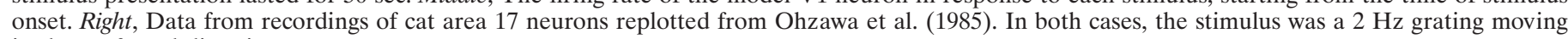
in the preferred direction.

tion to the high-contrast grating causes a DC hyperpolarization (Fig. 9A-D) in agreement with the results of Carandini and Ferster (1997). However, the amplitude of the oscillations in response to the $5 \%$ (Fig. $9 B$ ) and $100 \%$ (Fig. 9C) contrast stimuli is clearly smaller for high-contrast adaptation than for lowcontrast adaptation, an effect not seen by Carandini and Ferster (1997) in the majority of cells they recorded. In Figure $9 A-C$, action potentials have been blocked in the model to show the membrane potential oscillations without the spiking nonlinearity. Figure $9 D$ shows the membrane potential of the model under the same conditions used in Figure $9 C$ but with the model producing spikes every time the neuron rises to the threshold potential (for clarity, the spikes have been truncated in this figure). The spike generation mechanism in the model clips the membrane potential oscillation so that it does not rise above the action potential threshold. Clearly, if action potentials are not integrated into the potential in some way, spiking seriously distorts the picture and greatly reduces the amount by which contrast adaptation modifies the amplitude of membrane potential oscillations. The effects of spikes have been included in the data from the intracellular recorded membrane potentials by a method of averaging and interpolation (Carandini and Ferster, 1997), but, ideally, the results of the model should be compared with intracellular recordings with action potentials internally blocked. Nevertheless, as Carandini and Ferster (1997) have noted, the data seem to suggest the presence of a tonic component in the synaptic input that slowly depresses, and this is not present in the model we have described.

\section{DISCUSSION}

Simple cells exhibit nonlinear behavior in both the spatial and temporal domains, but the nature and source of these nonlinearities appear to be different. A large body of data suggests that spatial summation of subthreshold inputs to simple cells is linear and that the dominant source of nonlinearity in the spatial domain is the action potential threshold (Ferster, 1994). Nonlinear behavior in the time domain seems to be richer and difficult to explain as arising purely from a spiking threshold. Transient and sustained visual responses exhibit different types and degrees of nonlinear behavior. Transient responses show strong temporal nonlinearities, including enhancement of high-frequency components (Ikeda and Wright, 1975; Movshon et al., 1978; Kulikowski et al., 1979; Tolhurst et al., 1980). Sustained responses show more subtle temporal nonlinearities, in particular the failure of linear 

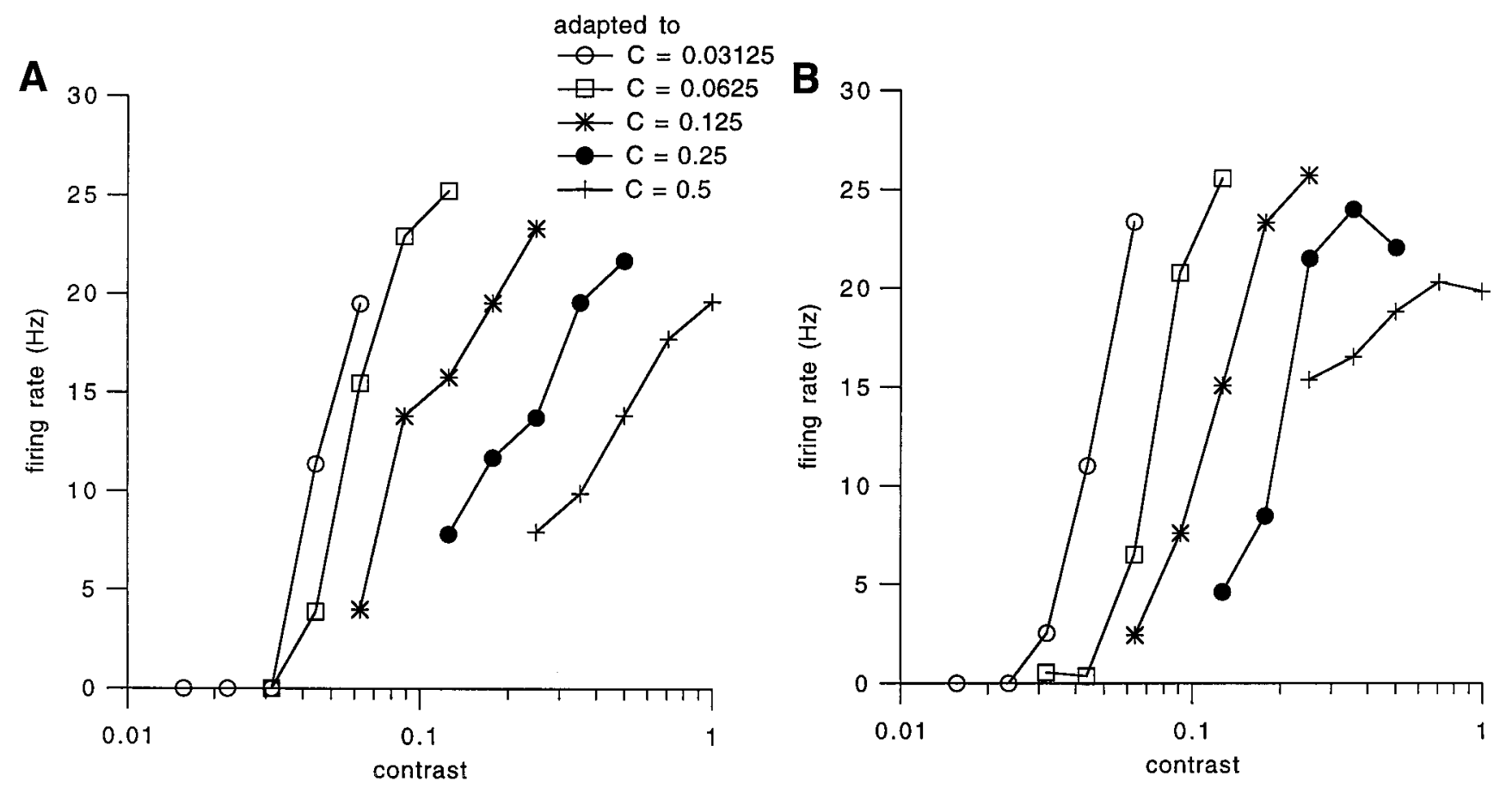

Figure 8. Contrast-response curves for different levels of adaptation. Each curve shows the response as a function of contrast after the neuron has been fully adapted to the level of contrast indicated. The stimulus was a $2 \mathrm{~Hz}$ grating moving in the preferred direction. $A$, Contrast-response curves of the model neuron. $B$, Contrast-response curves of a cat area 17 neuron redrawn from Ohzawa et al. (1985).

summation (Dean et al., 1982; Reid et al., 1992). We have found that the synaptic depression measured and modeled from experiments involving slices of primary visual cortex (Abbott et al., 1997; Varela et al., 1997) can account for nonlinear temporal effects in responses to both sustained and transient stimuli. Synaptic depression is particularly well suited to explain the marked differences between the responses to transient and periodic stimuli, including both the higher response frequency range and the increased response amplitude for transient stimuli.

Perceptual and single-unit studies have revealed the existence of sustained and transient channels in vision (for review, see Maunsell, 1987). Although a number of factors, including retinal and LGN cell type, contribute to this distinction, our studies indicate that differences in the degree and type of short-term synaptic plasticity can dramatically affect the temporal character of cortical responses.

Despite its extreme simplicity, our simple-cell model does a fairly good job of matching data on nonlinear temporal summation, direction selectivity as a function of frequency and contrast, and contrast adaptation. Results on the contrast dependence of response phase shifts suggest that synaptic depression may be a significant contributor to this effect (see also Priebe et al., 1997).

Previous models of directionally selective simple cells have used a variety of mechanisms to generate the necessary temporal phase shifts (Saul and Humphrey, 1990; Suarez et al., 1995; Maex and Orban, 1996). In these models, direction selectivity arises from a temporal delay combined with a spatial phase shift. In our model, direction selectivity arises from a phase advance. The intracellular recordings of Jagadeesh et al. (1993) shown in Figure 4 and the principal components extracted from these data by Kontsevich (1995) shown in Figure $3 B$ suggest that the more nonlinear component is phase advanced relative to the more linear (that is, sinusoidal) component. If the source of the non- linearity and the source of the phase shift are the same, as they are in our model, the phase shift must be an advance not a delay. A model based on a delay mechanism must use an approximately linear delay mechanism and then explain by a separate mechanism the nonlinear behavior in the other component. The intracellular recordings can potentially be explained by a single mechanism if the source of direction selectivity is a phase advance.

In the directionally selective model, we have adjusted the relative strengths of the depressing and nondepressing synapses so that their steady-state amplitude is approximately equal. As a result, the synaptic conductance parameter that sets the synaptic strength in the model was larger for depressing synapses than for nondepressing synapses. This is consistent with the assumption that these two sets of synapses differ in the probability of vesicle release. It is observed experimentally that depression is maximal for synapses with high release probabilities and decreases when the release probability is smaller (Atwood and Wojtowicsz, 1986; Zucker, 1989; Tsodyks and Markram, 1997; Varela et al., 1997). Because the synaptic strength parameter $g_{j}$ is proportional to release probability, this is exactly the type of relationship used in the directionally selective model.

The directionally selective model requires a correlation between the spatial phase of a particular afferent and the degree of depression at its synapse onto the V1 cell. Deficits in strobereared cats suggest that activity may play a role in establishing direction selectivity (Pasternak et al., 1985). The correlations needed in our model could arise from an activity-dependent learning rule that adjusts the degree of synaptic depression on the basis of the spatial phase of the input. Markram and Tsodyks (1996) have found that a long-term potentiation (LTP) paradigm that enhances the amplitude of postsynaptic currents in response to a single presynaptic spike also increases the degree of synaptic depression, so that the steady-state response to repetitive presyn- 

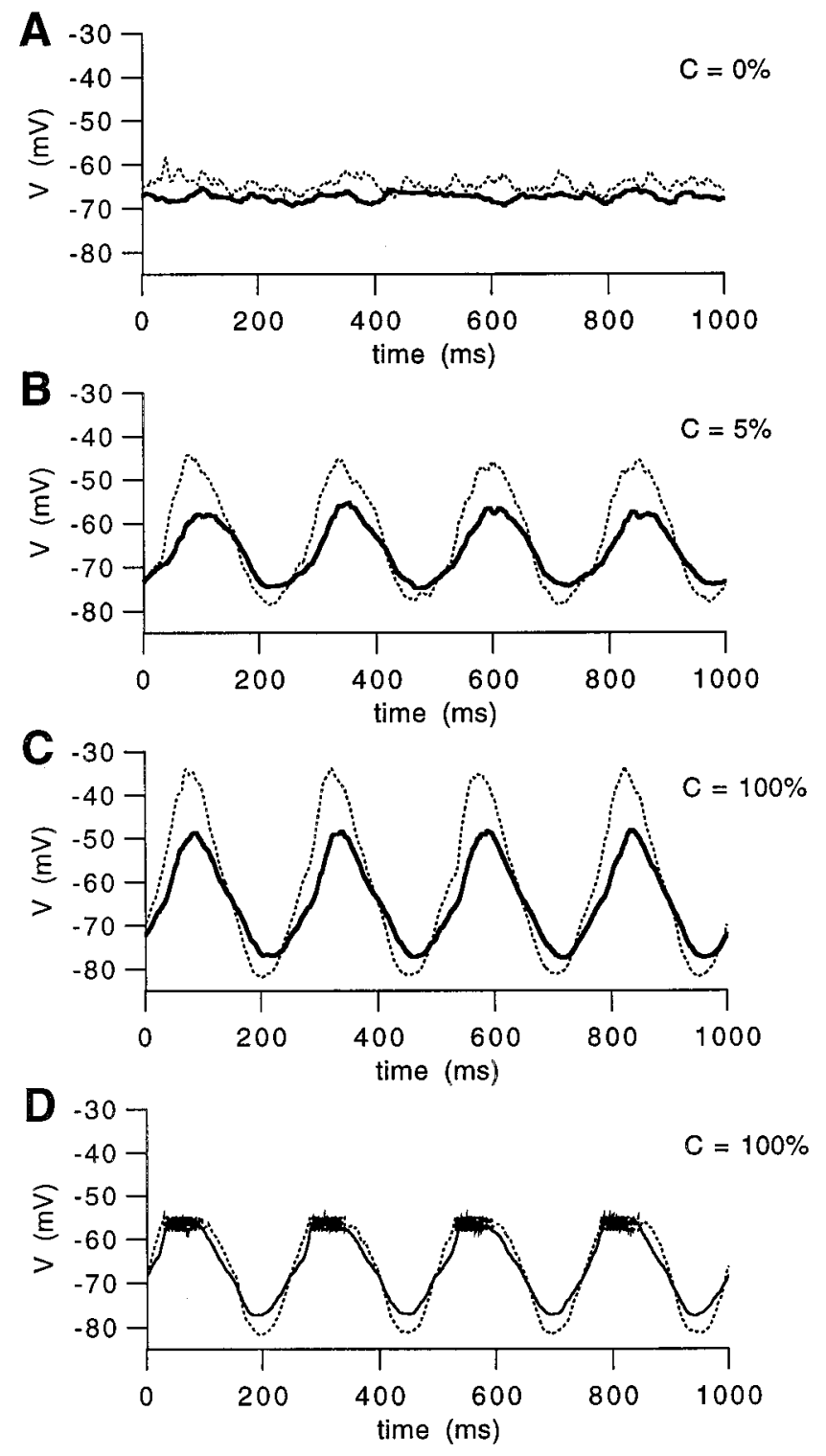

Figure 9. Membrane potential oscillations evoked by a $4 \mathrm{~Hz}$ oscillating grating at different contrast levels and for different degrees of adaptation. The solid curves show the response of the model V1 cell (with action potentials blocked) after prolonged exposure to an oscillating $100 \%$ contrast grating. For the dashed curves, the adapting stimulus was a 5\% grating. $A$, Response to a $0 \%$ grating. The different levels of contrast adaptation cause a DC shift in the membrane potential. $B$, Response to a $5 \%$ grating. $C$, Response to a $100 \%$ contrast grating. In $B$ and $C$, the different levels of contrast adaptation cause both a DC shift in the membrane potential and a change in the amplitude of the membrane potential oscillations. $D$, Same as $C$ but with the spike generation mechanism in the model activated. Spikes have been truncated to reveal the underlying subthreshold membrane potential more clearly.

aptic input remains unchanged. A form of LTP that targets the degree of synaptic depression is exactly what is needed for activity-dependent development of directional selectivity via synaptic depression. Modeling work on correlation-based activitydependent development (Miller, 1992) of direction selectivity in other models (Miura et al., 1995; Feidler et al., 1997) may provide the mechanism needed to correlate activity-dependent modification of the degree of depression with the spatial phase of a particular input, although this has not yet been verified.
Synaptic transmission at a synapse that exhibits depression depends on the relation of presynaptic activity to the activity that immediately preceded it. The presynaptic spiking pattern that produces the largest postsynaptic current is a period of silence followed by a high rate of activity. Visual neurons often respond most vigorously if the appearance of an optimal image is preceded by an "opposite" image, for example, contrast reversed. Recently Ringach et al. (1997) have measured reverse correlations for orientation tuning at short time intervals. Their data show this "reversal" effect; many neurons respond most vigorously if an optimally oriented image is preceded by an orthogonal orientation [the effects of synaptic depression on the dynamics of orientation tuning have been considered by O. Artun, H. Shouval, and L. Cooper (personal communication)]. Optimal stimuli that are anticorrelated in this way arise naturally from synaptic depression.

We have studied the idea that synaptic depression may also play an important role in contrast adaptation. The onset of synaptic depression depends on the number of presynaptic action potentials and thus is both rate and contrast dependent. Consistent with this, the magnitude and onset rate of adaptation increase as the temporal frequency of the visual stimulus increases (Maddess et al., 1988; Bonds, 1991; Nelson, 1991a). Pharmacological manipulations (Nelson, 1991b; McLean and Palmer, 1996) and intracellular recordings (Carandini and Ferster, 1997) support the hypothesis that contrast adaptation is caused by a reduction in excitatory synaptic drive. A number of experiments suggest a synaptic, rather than a cellular, adaptation mechanism. Contrast adaptation can occur locally within a portion of the receptive field of a cell (Marlin et al., 1991; Nelson, 1991a), the sensitivity of a neuron to a particular stimulus can be reduced without reducing its maximal firing rate (Ohzawa et al., 1985), and contrast adaptation can be induced by stimuli that do not cause the cortical neuron to fire (Vidyasagar, 1990; Geisler and Albrecht, 1992; Allison and Martin, 1997). On the other hand, recent results show that contrast adaptation can also be evoked by current injection without visual stimulation, suggesting that a cellular mechanism may also contribute (Sanchez-Vives et al., 1997).

We have constructed our model specifically to investigate the impact of synaptic depression and thus have tried to avoid other sources of temporal nonlinearity that would complicate the study of this particular mechanism. We are not proposing that all of the temporal response properties of cortical neurons arise solely from synaptic depression. Undoubtedly, neuronal adaptation and cortical circuit effects play a role in these phenomena. Some of the effects of the interplay between recurrent synaptic connections, cortical amplification (Douglas et al., 1995), and synaptic depression have been discussed by Todorov et al. (1997), Priebe et al. (1997), and Sen (1997). Interplay between the different forms of short-term plasticity seen in intracortical excitatory-excitatory, excitatory-inhibitory, and inhibitory-excitatory synapses, as well as the rich dynamics of recurrent neural circuits, will make the study of more realistically connected models both challenging and interesting.

The most direct way to test our model experimentally is to modify synaptic depression in vivo. Recently, we and others have shown that manipulations that diminish transmitter release also diminish synaptic depression in slices (Gil et al., 1997; Tsodyks and Markram, 1997; Varela et al., 1997). These manipulations include reducing extracellular calcium, applying neuromodulators including adenosine and acetylcholine, and activating presynaptic GABA-B receptors. According to our model, reducing 
depression should make V1 responses more linear in the temporal domain, and the response properties that we have attributed to depression, such as enhancement of transient and reduction of steady-state responses, nonlinear temporal summation, contrastdependent phase shifts, directional selectivity, and contrast adaptation, should be altered. Unfortunately, manipulations that modify transmitter release do not target synaptic depression exclusively; they also affect response amplitude and synaptic facilitation. Spiking threshold effects in extracellular recordings can make it difficult to untangle these combined modulations. Nevertheless, the model makes clear predictions about the impact that modifying synaptic depression will have on visual responses, and with sufficient pharmacological specificity and clever data analysis, it should be possible to test them.

\section{REFERENCES}

Abbott LF, Sen K, Varela JA, Nelson SB (1997) Synaptic depression and cortical gain control. Science 275:220-224.

Adelson EH, Bergen JR (1985) Spatiotemporal energy models for the perception of motion. J Opt Soc Am A2:284-299.

Ahmed B, Allison JD, Douglas RJ, Martin KAC (1997) An intracellular study of the contrast-dependence of neuronal activity in cat visual cortex. Cereb Cortex 7:559-570.

Albrecht DG, Hamilton DB (1982) Striate cortex of monkey and cat: contrast response function. J Neurophysiol 48:217-237.

Albrecht DG, Farrar SB, Hamilton DB (1984) Spatial contrast adaptation characteristics of neurones recorded in the cat's visual cortex. J Physiol (Lond) 347:713-739.

Allison JD, Martin KAC (1997) Contrast adaptation produced by null direction and cross orientation stimulation of neurons in cat visual cortex. Soc Neurosci Abstr 23:454.

Atwood HL, Wojtowicsz JM (1986) Short-term and long-term plasticity and physiological differentiation of crustacean motor synapses. Int Rev Neurobiol 28:275-362.

Barlow H, Levick R (1965) The mechanism of directional selectivity in the rabbit's retina. J Physiol (Lond) 173:477-504.

Bonds AB (1991) Temporal dynamics of contrast gain in single cells of the cat striate cortex. Vis Neurosci 6:239-255.

Borst A, Egelhaaf M (1989) Principles of visual motion detection. Trends Neurosci 12:297-306.

Carandini M, Ferster D (1997) A tonic hyperpolarization underlying contrast adaptation in cat visual cortex. Science 276:949-952.

Carandini M, Heeger DJ (1994) Summation and division by neurons in primate visual cortex. Science 264:1333-1336.

Carandini M, Heeger DJ, Movshon JA (1998) Linearity and gain control in V1 simple cells. In: Cerebral cortex, Vol XII (Jones EG, Ulinski PS, eds). New York: Plenum, in press.

Castro-Alamancos M, Connors B (1996) Spatiotemporal properties of short-term plasticity in sensorimotor thalamocortical pathways of the rat. J Neurosci 16:2767-2779.

Cheng H, Chino YM, Smith EL, Hamamoto J, Yoshida K (1995) Transfer characteristics of lateral geniculate nucleus $X$ neurons in the cat: effects of spatial frequency and contrast. J Neurophysiol 74:2548-2557.

Dean AF, Tolhurst DJ (1986) Factors influencing the temporal phase of response to bar and grating stimuli for simple cells in the cat striate cortex. Exp Brain Res 62:143-151.

Dean AF, Tolhurst DJ, Walker NS (1982) Non-linear temporal summation by simple cells in cat striate cortex demonstrated by failure of superposition. Exp Brain Res 45:456-458.

Deisz R, Prince D (1989) Frequency-dependent depression of inhibition in guinea-pig neocortex in vitro by $\mathrm{GABA}_{\mathrm{B}}$ receptor feedback on GABA release. J Physiol (Lond) 412:513.

Douglas RJ, Koch C, Mahowald M, Martin KAC, Suarez HH (1995) Recurrent excitation in neocortical circuits. Science 269:981-985.

Feidler JC, Saul AB, Murthy A, Humphrey AL (1997) Hebbian learning and the development of direction selectivity: the role of geniculate response timings. Network 8:192-214.

Ferster D (1994) Linearity of synaptic interactions in the assembly of receptive fields in cat visual cortex. Curr Opin Neurobiol 4:563-568.

Finlayson PG, Cynader MS (1995) Synaptic depression in visual cortex tissue slices: an in vitro model for cortical neuron adaptation. Exp Brain Res 106:145-155.
Geisler WS, Albrecht DG (1992) Cortical neurons: isolation of contrast gain control. Vision Res 32:1409-1410.

Giaschi D, Douglas R, Marlin S, Cynader M (1993) The time course of direction-selective adaptation in simple and complex cells in cat striate cortex. J Neurophysiol 70:2024-2034.

Gil Z, Amitai Y, Castro MA, Connors BW (1997) Differential regulation of neocortical synapses by neuromodulators and activity. Neuron 19:679-686.

Grossberg S (1984) Some psychophysiological and pharmacological correlates of a developmental, cognitive, and motivational theory. In: Brain and information: event related potentials (Karrer R, Cohen J, Tueting P, eds), pp 58-142. New York: NY Acad Sci.

Hamilton DB, Albrecht DG, Geisler WS (1989) Visual cortical receptive fields in monkey and cat: spatial and temporal phase transfer function. Vision Res 29:1285-1308.

Hawken MJ, Shapley RM, Grosof DH (1996) Temporal-frequency selectivity in monkey visual cortex. Vis Neurosci 13:477-492.

Heeger DJ (1992) Normalization of cell responses in cat striate cortex. Vis Neurosci 9:181-198.

Heeger DJ (1993) Modeling simple-cell direction selectivity with normalized, half-squared, linear operators. J Neurophysiol 70:1885-1898.

Hubel DH, Wiesel TN (1962) Receptive fields, binocular interaction and functional architecture in the cat's visual cortex. J Physiol (Lond) 160:106-154

Ho WA, Berkley MA (1988) Evoked potential estimates of the time course of adaptation and recovery to counterphase grating. Vision Res 28:1287-1296.

Ikeda H, Wright MJ (1975) The relationship between the "sustainedtransient" and the "simple-complex" classifications of neurones in area 17 of the cat. J Physiol (Lond) 244:58P-59P.

Jagadeesh B, Wheat HS, Ferster D (1993) Linearity of summation of synaptic potentials underlying direction selectivity in simple cells of the cat visual cortex. Science 262:1901-1904.

Kontsevich LL (1995) The nature of the inputs to cortical motion detectors. Vision Res 35:2785-2793.

Krausz HI, Friesen WO (1977) The analysis of nonlinear synaptic transmission. J Gen Physiol 70:243-265.

Kulikowski JJ, Bishop PO, Kato H (1979) Sustained and transient responses by cat striate cells to stationary flashing light and dark bars. Brain Res 170:362-367.

Liley AW, North KAK (1952) An electrical investigation of effects of repetitive stimulation on mammalian neuromuscular junction. J Neurophysiol 16:509-527.

Maddess T, McCourt ME, Blakeslee B, Cunningham RB (1988) Factors governing the adaptation of cells in area- 17 of the cat visual cortex. Biol Cybern 59:229-236.

Maex R, Orban GA (1992) A model circuit for cortical temporal lowpass filtering. Neural Comput 4:932-945.

Maex R, Orban GA (1996) Model circuit of spiking neurons generating directional selectivity in simple cells. J Neurophysiol 75:1515-1545.

Magleby KL, Zengel JE (1975) A quantitative description of tetanic and post-tetanic potentiation of transmitter release at the frog neuromuscular junction. J Physiol (Lond) 245:183-208.

Markram H, Tsodyks MV (1996) Redistribution of synaptic efficacy between neocortical pyramidal neurones. Nature 382:807-809.

Marlin SG, Douglas RM, Cynader MS (1991) Position-specific adaptation in simple cell receptive fields of the cat striate cortex. J Neurophysiol 66:1769-1784.

Mastronarde DN (1987) Two classes of single-input x-cells in cat lateral geniculate nucleus. Cat lateral geniculate nucleus. I. Receptive-field properties and classification of cells. J Neurophysiol 57:357-380.

Maunsell JHR (1987) Physiological evidence for two visual subsystems. In: Matters of intelligence (Vaina LM, ed), pp 59-87. New York: Reidel.

McLean J, Palmer LA (1996) Contrast adaptation and excitatory amino acid receptors in cat striate cortex. Vis Neurosci 13:1069-1087.

Miller KD (1992) Models of activity-dependent neural development. Semin Neurosci 4:61-73.

Miura K, Kurata K, Nagano T (1995) Self-organization of the velocity selectivity of a directionally selective neural network. Biol Cybern 73:401-407.

Movshon JA, Lennie P (1979) Pattern-selective adaptation in visual cortical neurones. Nature 278:850-852.

Movshon JA, Thompson ID, Tolhurst DJ (1978) Spatial and temporal 
contrast sensitivity of neurones in areas 17 and 18 of the cat's visual cortex. J Physiol (Lond) 283:101-120.

Nelson SB (1991a) Temporal interactions in the cat visual system. I. Orientation-selective suppression in the visual cortex. J Neurosci 11:344-356.

Nelson SB (1991b) Temporal interactions in the cat visual system. III. Pharmacological studies of cortical suppression suggest a presynaptic mechanism. J Neurosci 11:369-380.

Nelson SB, Varela JA, Sen K, Abbott LF (1997) Functional significance of synaptic depression between cortical neurons. In: Computational neuroscience, trends in research (Bower JM, ed), pp 429-434. New York: Plenum.

Ohzawa I, Sclar G, Freeman RD (1985) Contrast gain control in the cat's visual system. J Neurophysiol 54:652-667.

Orban GA, Hoffmann KP, Duysens J (1985) Velocity selectivity in the cat visual system. I. Responses of LGN cells to moving bar stimuli: a comparison with cortical areas 17 and 18. J Neurophysiol 54:1026-1049.

Orban GA, Kennedy H, Bullier J (1986) Velocity sensitivity and direction selectivity of neurons in areas V1 and V2 of the monkey: influence of eccentricity. J Neurophysiol 56:462-480.

Pasternak T, Schumer RA, Grizzi MS, Movshon JA (1985) Abolition of visual cortical direction selectivity affects visual behavior in cats. Exp Brain Res 61:214-217.

Priebe NJ, Kayser AS, Krukowski AE, Miller KD (1997) A model of simple cell orientation tuning: the role of synaptic depression. Soc Neurosci Abstr 23:2061.

Reid RC, Soodak RE, Shapley RM (1991) Direction selectivity and spatiotemporal structure of receptive fields of simple cells in cat striate cortex. J Neurophysiol 66:505-529.

Reid RC, Victor JD, Shapley RM (1992) Broadband temporal stimuli decrease the integration time of neurons in cat striate cortex. Vis Neurosci 9:39-45.

Ringach DL, Hawken MJ, Shapley R (1997) Dynamics of orientation tuning in macaque primary visual cortex. Nature 387:281-284.

Sanchez-Vives MV, Nowak LG, McCormick DA (1997) Cellular and network mechanisms generating adaptation to contrast in the visual cortex: an in vivo and in vitro study. Soc Neurosci Abstr 23:1944.

Saul AB, Humphrey AL (1992) Spatial and temporal properties of lagged and nonlagged cells in the cat lateral geniculate nucleus. J Neurophysiol 68:1190-1208.

Saul AB, Humphrey AL (1992) Temporal-frequency tuning of direction selectivity in cat visual cortex. Vis Neurosci 8:365-372.

Sen K (1997) The temporal dynamics of synapses and synaptic decoding. PhD Thesis, Brandeis University.

Sen K, Jorge-Rivera JC, Marder E, Abbott LF (1996) Decoding synapses. J Neurosci 16:6307-6318.

Shaw C, Teyler TJ (1982) The neural circuitry of the neocortex examined in the in vitro brain slice preparation. Brain Res 243:35-47.

Skottun BC, Bradley A, Ramoa AS (1986) Effect of contrast on spatial frequency tuning of neurones in area 17 of cat's visual cortex. Exp Brain Res 63:431-435.

Smith A, Snowden R (1994) Visual detection of motion. London: Academic.
Song S, Varela JA, Abbott LF, Nelson SB (1997) A quantitative description of synaptic depression at monosynaptic inhibitory inputs to visual cortical pyramidal neurons. Soc Neurosci Abstr 23:2362.

Stratford KJ, Tarczy-Hornuch K, Martin KAC, Bannister NJ, Jack JJB (1996) Excitatory synaptic inputs to spiny stellate cells in cat visual cortex. Nature 382:258-261.

Suarez H, Koch C, Douglas R (1995) Modeling direction selectivity of simple cells in striate visual cortex within the framework of the canonical microcircuit. J Neurosci 15:6700-6719.

Thomson AM, Deuchars J (1994) Temporal and spatial properties of local circuits in neocortex. Trends Neurosci 17:119-126.

Thomson AM, West DC (1993) Fluctuations in pyramid-pyramid excitatory postsynaptic potentials modified by presynaptic firing pattern and postsynaptic membrane potential using paired intracellular recordings in rat neocortex. Neuroscience 54:329-346.

Thomson AM, Deuchars J, West DC (1993) Large, deep layer pyramidpyramid single axon EPSPs in slices of rat motor cortex display paired pulse and frequency-dependent depression, mediated presynaptically and self-facilitation, mediated postsynaptically. J Neurophysiol 70:2354-2369.

Todorov EV, Siapas AG, Somers DC, Nelson SB (1997) Modeling visual cortical contrast adaptation effects. In: Computational neuroscience, trends in research (Bower JM, ed), pp 525-531. New York: Plenum.

Tolhurst DJ, Dean AF (1991) Evaluation of a linear model of directional selectivity in simple cells of the cats striate cortex. Vis Neurosci 6:421-428.

Tolhurst DJ, Heeger DJ (1997) Contrast normalization and a linear model for the directional selectivity of simple cells in cat striate cortex. Vis Neurosci 14:19-25.

Tolhurst DJ, Walker NS, Thompson ID, Dean AF (1980) Nonlinearities of temporal summation in neurones in area 17 of the cat. Exp Brain Res 38:431-435.

Tsodyks MV, Markram H (1997) The neural code between neocortical pyramidal neurons depends on neurotransmitter release probability. Proc Natl Acad Sci USA 94:719-723.

Varela J, Sen K, Gibson J, Fost J, Abbott LF, Nelson SB (1997) A quantitative description of short-term plasticity at excitatory synapses in layer 2/3 of rat primary visual cortex. J Neurosci 17:7926-7940.

Vidyasagar TR (1990) Pattern adaptation in cat visual cortex is a cooperative phenomenon. Neuroscience 36:175-179.

Watson AB, Ahumada AJ (1985) Model of human visual-motion sensing. J Opt Soc Am A2:322-342.

Wörgötter F, Koch C (1991) A detailed model of the primary visual pathway in the cat: comparison of afferent excitatory and intracortical inhibitory connection schemes for orientation selectivity. J Neurosci 11:1959-1979.

Zengel JE, Magleby KL (1982) Augmentation and facilitation of transmitter release. J Gen Physiol 80:583-611.

Zucker RS (1989) Short-term synaptic plasticity. Annu Rev Neurosci 12:13-31. 